\title{
Geometric polarimetry - part II: the Antenna Height Spinor and the Bistatic Scattering Matrix
}

Article

Accepted Version

Bebbington, D. and Carrea, L. (2017) Geometric polarimetry part II: the Antenna Height Spinor and the Bistatic Scattering Matrix. IEEE Transaction on Geoscience and Remote Sensing, 55 (8). pp. 4296-4313. ISSN 1558-0644 doi: https://doi.org/10.1109/TGRS.2017.2690972 Available at https://centaur.reading.ac.uk/70124/

It is advisable to refer to the publisher's version if you intend to cite from the work. See Guidance on citing.

To link to this article DOI: http://dx.doi.org/10.1109/TGRS.2017.2690972

Publisher: IEEE

All outputs in CentAUR are protected by Intellectual Property Rights law, including copyright law. Copyright and IPR is retained by the creators or other copyright holders. Terms and conditions for use of this material are defined in the End User Agreement.

www.reading.ac.uk/centaur 
Central Archive at the University of Reading

Reading's research outputs online 


\title{
Geometric Polarimetry - Part II: the Antenna Height Spinor and the Bistatic Scattering Matrix
}

\author{
David Bebbington and Laura Carrea
}

\begin{abstract}
This paper completes the fundamental development of the basic coherent entities in Radar Polarimetry for coherent reciprocal scattering involving polarized wave states, antenna states and scattering matrices. The concept of antenna polarization states as contravariant spinors is validated from fundamental principles in terms of Schelkunoff's reaction theorem and the Lorentz reciprocity theorem. In the general bistatic case polarization states of different wavevectors must be related by the linear scattering matrix. It is shown that the relationship can be expressed geometrically, and that each scattering matrix has a unique complex scalar invariant characterising a homographic mapping relating pairs of transmit/receive states for which the scattering amplitude vanishes. We show how the scalar invariant is related to the properties of the bistatic Huynen fork in both its conventional form and according to a new definition. Results are presented illustrating the invariant $k$ for a range of spheroidal Rayleigh scatterers.
\end{abstract}

Index Terms-spinors, antenna height, reciprocity, bistatic invariants.

\section{INTRODUCTION}

Modern polarimetric radars are capable of high bandwidth coherent processing and recording of $I, Q$ data. Although multilook averaging in SAR [1] for natural targets and multisampling in weather radar [2] involve partially coherent averaging techniques there is now more than ever a need to understand fully both real and abstract geometric relationships in coherent scattering. The use of basis or geometric transformations, in solving polarimetric problems continues to feature in the literature [3], [4] [5]. In recent years there has been an increase in interest in polarimetric bistatic scattering as technical capabilities for multi-platform coherent systems (e.g. [6]) have improved.

Although, as yet, remote sensing platforms capable of full polarimetric bistatic measurement are not widely available, measurements of bistatic scattering are increasingly being seen as having potential in relation to sensing crops and other vegetation. Scattering from soil is often a dominant element in the return from vegetated surfaces, and owing to the uncertainties in moisture and roughness it remains a challenging problem to model surface contributions from backscattering alone. Guerriero et al [7] report simulations for corn fields in which the potential for obtaining crop parameters is claimed. Carreno-Luego et al [8] have presented measurements of scattering of GNSS-R signals received bistatically

This work was supported by the Marie Curie Research Training Network AMPER (Contract number HPRN-CT-2002-00205).

D. Bebbington is with the Centre for Remote Sensing \& Environmetrics, School of Computer Science and Electronic Engineering, University of Essex, U.K. (email: david@essex.ac.uk); L. Carrea is with the Department of Meteorology, University of Reading, U.K. (email: 1.carrea@ reading.ac.uk). and multistatically from a stratospheric platform over boreal forests which showed that there are important polarimetric signatures involving the canopy-soil interaction. Santalla del Rio et al [9] have recently presented an analytical approach to modelling the coherent bistatic scattering from vegetation components modelled as finite cylinders.

Even so, in recent years there has not been a significant development of a bistatic scattering theoretical framework such as occurred for the backscattering case around the turn of the century [10], [11].

Davidowitz and Boerner [12] produced a classic study on bistatic scattering which considered how far the established techniques for characterizing backscatter could be extended to the bistatic case. Here, the classic 'Huynen fork' [13] concept can still, in a sense, be considered, although the four characteristic polarizations are no longer coplanar. More recent works of Germond [14], [15], Cloude [16], Bombrun [17], [18], and Titin-Schneider [19], [20] were concerned with how one can derive intrinsic, basis-independent descriptors of targets, and characterize their invariant properties. Common to several of these cited papers is a standard matrix based approach, where, in the absence of meaningful eigen-methods, singular value decomposition (SVD) methods are used to determine polarizations for which total power is maximal or stationary as in a saddle point. In this paper, we take the reverse path from earlier authors, by starting with the general bistatic case, rather than extending from the specialized backscatter case. In Sec. VI we will be considering how the geometric approach motivated by the spinor representation leads to a new complex invariant for a bistatic scatterer. We find that, at least for a class of electrically small scatterers, the invariant is sensitive both in amplitude and phase to shape and inclination of the scatterer. Monostatic scattering, which involves greater symmetries, has yet further interesting features that can be revealed using geometric methods that space does not permit to be presented in the present paper. In the first paper [21] of the projected formal series on Geometric Polarimetry we undertook the most detailed investigation to date into how spinor algebra can be used in a rigorous way to represent electromagnetic polarization. This paper is a direct continuation, and the reader will be referred back to it for many of the fundamental developments that will be required here. The most significant features to emerge in [21] were that spinors should be considered to represent complex coherent wave states geometrically not as vectors in the tradition of Jones vectors [22] but rather as generating lines on the Poincaré sphere via a direct construction in complex projective space. As we progress through the foundations of polarimetry it will become 
increasingly clearer that this concept underpins the inherent geometric connection between all polarimetric descriptors. The fundamental reason for this is that under any linear transformation of the coherent polarization states the Poincaré sphere itself is an invariant object. References [23] and [24] are the well known standard texts that explain respectively practically all that needs to be known about $2-$ spinor calculus and projective geometry using homogeneous coordinates. It is the fusion of concepts from these two domains that has given rise to Geometric Polarimetry. In an earlier paper [25] we were able to clarify the status of Graves' congruential rule for basis transformation in the backscatter alignment (BSA) case against the hitherto quite widely accepted consimilarity transformation [26]. In particular we noted that the widely held notion that counterpropagating waves of the 'same' polarization state are to be considered as mutually conjugate is invalid in the wider physical context of absorbing media. More generally, in the polarimetric context such a misrepresentation, geometrically, obscures the bigger picture in which a more satisfactory framework can be constructed, and which leads to clearer formulations of problems. Such problems can then be solved using more mainstream mathematical techniques for which there is a much larger literature, and well established robust numerical techniques are available. A second significant result of [25] was that separate representations for the coherent electromagnetic field and the complex antenna height vector are required. It came to be regarded as unquestionable that the field transmitted by an antenna state is identical up to scale factor with the complex antenna height vector. However, it was only through the rigorous application of spinor algebra that it became possible to show that the spin spaces in which these two kinds of object are to be represented must be different if the algebraic machinery for unitary basis change is to be valid. This distinction is fundamental to much of the further issues that we plan to present both in this paper and further publications. In particular we will regard the voltage equation (c.f. [27]) for a covariant scatterer $S_{A B}$, and contravariant receive and transmit antenna height spinors $\xi^{A}, \eta^{A}$,

$$
V=\left(\frac{I Z_{0}}{\lambda r^{2}}\right) \eta^{A} S_{A B} \xi^{B}
$$

as the primary representation of the scattering process. In (1), $Z_{0}$ is the free space impedance, $\lambda$ is the wavelength, $r$ is the distance from the antenna $(r>>\lambda)$ and $I$ is the driving current. In future we will absorb the scalar factor in brackets involving dependency on range and wavelength into $S_{A B}$, and assume a unit driving current, $I$. Many polarimetrists [20], [28] perhaps still favour the form of scattering equation that relates transmitted and received fields, as in the formulation of Graves [29], as it may seem 'more fundamental'. We would say that the covariant form, $S_{A B}$, is what is actually measured, and the field form can only be deduced. These points reflect individual preferences, however, a practical argument against the field form is that it requires states to be referenced to different spin spaces for the transmitted and received waves, which Graves' directional waves are equivalent to. The relation (1) is really the one that is true to the spirit of the backscatter alignment convention because it refers to the transmit and receive an- tennas that are co-located and expressed with reference to the same frame. Not all polarimetrists are adherents to BSA [30] but for those who are, (1) should be the prime justification. The further advantage of (1) is that it is possible for the most part to avoid having to deal with both kinds of polarization spinors, and refer only to the antenna polarization. In this way one avoids confusion between the two kinds of polarization states when expressed in terms of numerical components but without symbolic indices, to show the type. Whilst in [25] we deduced the spinor character of the antenna height as contravariant, space did not permit an explicit derivation. In [21] we concentrated exclusively on representations of wave state. Given that it has now been shown that wave states have covariant spinor representations, we are now in a position to give a similar derivation from first principles of the antenna height spinor. With this in place, the validity of (1) will be cemented and it will be shown how this representation can be exploited to improve understanding of fundamental polarimetric relationships using geometric principles. The plan of the remainder of the paper is as follows. In section IV we develop a rigorous derivation of antenna height spinors using results from [21] and applying Schelkunoff's reaction theorem [31], [32]. In section VI, we develop the geometrical properties of the scattering matrix, seen as a bilinear form as in (1), while we devote section VIII to a modified definition of the Huynen polarization fork for the general bistatic case. In section IX we present the simulations of bistatic scattering for different geometries of two types of scatterers, spheroids and composite scatterers. The results show how the invariant $k$ which characterises each scattering matrix is sensitive to shape and orientation of the scatterers. A comparison of the invariant with the SVD is also given.

\section{MOTIVATION FOR REPLACING JONES VECTORS WITH SPINORS}

Traditionally, coherent polarimetric relations have been expressed in terms of Jones vectors. Essentially these are considered to be two-dimensional complex vectors in the plane parallel to the wavefront. Formally, these have been considered as complex extensions to Euclidean vectors. In this sense, Euclidean isometries such as rotation can be applied without introducing any problems, since real and imaginary parts transform independently. A particular bonus of the Jones calculus was that Jones vectors appeared also to transform under unitary basis transformations. It might be said that is where problems began to arise in polarimetry because the two concepts are not formally consistent: Jones vectors effectively acquired a dual identity as both vectors and spinors, which are the carriers of the special unitary group, SU(2). In [21] this problem was resolved by identifying the descriptors that transform unitarily, and properly, as spinors, while Jones vectors remain properly as complex vectors. For any fixed wave propagation direction there is a 1:1 mapping between the two, which in some cases appears numerically trivial, but is inherently non-trivial, since spinors correspond geometrically to the complex generators of the Poincare sphere, rather than to vectors. Fundamentally, the equivalence of all generators on a 
quadric surface means that one can handle unitary polarization representations uniformly for any direction of radiation. One of the further problems of using Jones vector representations for polarization is that the conventional Euclidean representation in Cartesian coordinates does not allow formal distinction between pure polarization states of the field (Jones vectors, proper, as introduced in optics) and polarization states of the antenna height that were introduced in radar [33], [34]. That there must be a difference is seen in the literature (e.g. [35]) where the antenna height vector is subjected to a different basis transformation from that of the wave state. The formal reasons for this became rather clouded by the issue of wave reversal, the backscatter alignment convention [35], [36], [37], [38], [39], [40] and the introduction of the consimilarity concept [26], which was only recently resolved in [25]. It can now be seen to be attributable to the fact that the field must be viewed as a covariant vector while the antenna height is a contravariant vector. This distinction between different types of vectors does not show up in conventional Cartesian vector and tensor analysis because the Euclidean metric tensor is numerically equal to the identity matrix, and therefore appears to be redundant. For spinors, however, the invariant metric is the unit skew matrix [23],

$$
\varepsilon_{A B}=\varepsilon^{A B}=\left(\begin{array}{rr}
0 & 1 \\
-1 & 0
\end{array}\right),
$$

which cannot be neglected. Consequently, the standard notation for spinors naturally distinguishes between covariant and contravariant types. As will be seen, assigning wave states to covariant spinors and antenna states to contravariant spinors reflects the difference in their basis transformation rules. In fact, covariant spinors are subject to inverse basis transformation with respect to contravariant spinors. Consequently, using spinors to represent the two types of polarization introduces a formal requirement which provides consistency and the necessary basis invariance.

From a practical point of view, it may now seem potentially confusing to have two different kinds of polarization, especially if the spinors are expressed in purely numerical form without the helpful indexing. In this approach we remain faithful to the polarimetric tradition that the state of polarization of the wave emitted, or maximally received is the same as that of the antenna; but since field and wave states are of different index type they cannot be said to be equal. One way to minimise any confusion is to avoid, where possible, using both at the same time. From an empirical and practical point of view, at least, there is much to be said for concentrating on antenna states alone. In fact, it is probably very rarely the case, in remote sensing analysis that the field as well as the antenna representation would both require to be explicitly referred to. For example, to measure a scattering matrix, what is performed operationally reduces in principle to measuring the receive voltage for two independent receive states, for two successive transmit states. One can then deduce what the fields would have been, if necessary. So, although, traditionally, scattering matrices have been expressed as operators on the incident field to produce the scattered field in the chosen scattered direction, this is not what is directly measured. It turns out, perhaps surprisingly, that the geometrical properties of scattering are easier to capture if we take the operational view as the primary description.

To motivate the geometrical techniques which carry forward the programme of Geometric Polarimetry it may prove useful to review some of the fundamental algebraic and geometric operations that complement traditional matrix calculus. Traditionally, matrix calculus consists of transforming one coordinate vector into another, and in polarimetry mainly it is dominated by eigenvalue/eigenvector analysis. In many branches of physics and engineering it is often more useful to consider multilinear forms which produce some scalar quantity as a function of one or more input vectors. In particular, bilinear or quadratic forms take a pair of vector-like inputs, and evaluate to a scalar. Such forms frequently have geometric representations, which not only provide an aid to visualization, but provide a guide for analysis of special cases.

\section{BILINEAR AND QUADRATIC FORMS}

In any scattering experiment with fixed geometry, the received voltage at the terminals of an antenna is a linear function of the incident field, if the antenna polarization is fixed. Since the incident field is linearly related to the transmit antenna, one can say equivalently that the received voltage is a linear function of the transmit antenna polarization state (assumed to be driven by a fixed current). Conversely, when the transmit state is fixed, the received voltage is a linear function of the receive antenna polarization state. Thus, the received voltage for a fixed scatterer is said to be a bilinear function of the transmit and receive polarization states. The operator which acts on the pair of antenna states and which characterizes the scatterer is therefore said to be a bilinear form. Superficially this appears to be a 'matrix' but the key reason for distinguishing it from a conventional matrix is that it is better thought of not as transforming fields but representing the empirical results of 'testing' the scatterer using variable polarizations. There is a further benefit from this approach in putting both antennas on an equal footing. In the special case of monostatic scattering and when reciprocity holds, it is immaterial which antenna transmits and which receives, so the bilinear form is said to be symmetric; symmetry of the bilinear matrix that represents the form follows automatically from this, which explains the symmetry of the backscatter matrix without any problems relating to wave reversal. In a further specialization in which the transmit and receive antennas are also identical one speaks of a quadratic form. In other words, the co-polar voltage is a quadratic form in the antenna state. Quadratic forms occupy a special place in applied mathematics. It may be useful to consider first analogies where only real vectors figure, a good example being the central moment of inertia tensor which relates rotational energy of a body to its angular velocity as a quadratic form. The quadratic form can be characterised geometrically as an ellipsoid, showing the contour of rotational energy as function of the angular velocity, whose principal axes represent the directions for which angular momentum and angular velocity are parallel. For example an ideal dumb-bell or rotor with axis 
along the $\mathrm{z}$-axis and two unit masses symmetrically disposed one unit from the origin has an inertia tensor of the form,

$$
I_{i j}=\left(\begin{array}{ccc}
1 & 0 & 0 \\
0 & 1 & 0 \\
0 & 0 & 0
\end{array}\right)
$$

The rotational energy $T$ and angular momentum $p_{i}$ are obtained from the angular velocity vector $\omega^{i}$ as,

$$
T=\frac{1}{2} \omega^{i} I_{i j} \omega^{j}, \quad p_{i}=\frac{\partial}{\partial \omega^{i}} T=I_{i j} \omega^{j},
$$

where the Einstein summation convention is used. In the general case, the direction of the angular momentum is determined as orthogonal to the tangent plane corresponding to the radial angular velocity. In the case of the rotor, the ellipsoid degenerates to a disc, since there is no rotational energy if the angular velocity is aligned with its axis.

This relationship also illustrates that angular momentum is a different type of vector from angular velocity, being of covariant type. Such distinctions are rarely mentioned explicitly when using Euclidean geometry, as they are most often disguised by the practice of constructing vectors that are orthogonal to surfaces or surface elements. The analysis of the moment of inertia tensor is in many ways similar to that of covariance matrices. The central moment of inertia tensor of any body can be decomposed into three orthogonal rotors. There are of course many bodies that map to the same decomposition.

The geometric interpretations of mechanics have their analogues in polarimetry where as was shown in [21] coherent plane electromagnetic waves can be represented by a covariant spinor. Correspondingly, an antenna state must be considered as a contravariant vector. The distinction arises because antennas are characterised by their effective height (in units of metres, say), while electric field strengths are measured in volts per metre. If, for example, one were to change the unit of measurement to centimetres, then the numerical value of the effective height of an antenna (in $\mathrm{cm}$ ) would increase while the numerical value of the field (in $\mathrm{V} / \mathrm{cm}$ ) would correspondingly decrease. The key point is that the inner product between these two quantities must be invariant whatever the units and whatever the coordinate system involved. The above example shows in a very simple way that the algebraic relations are preserved not only for Euclidean transformations.

\section{DERIVATION OF THE ANTENNA POLARIZATION SPINOR}

Just as in [21] we showed that the polarization state of a plane electromagnetic wave can be represented not only in the form of a tensor, or field vector potential, but also as a covariant spinor, so, in order to operate on spinor states to obtain received voltage, there has to be a contravariant polarization state spinor representing the antenna. Then one can write the fundamental voltage equation,

$$
V=\psi_{A} \eta^{A}=\left(\begin{array}{l}
\psi_{0} \\
\psi_{1}
\end{array}\right) \cdot\left(\begin{array}{l}
\eta^{0} \\
\eta^{1}
\end{array}\right)=\psi_{0} \eta^{0}+\psi_{1} \eta^{1},
$$

where $\psi_{A}$ is the wave spinor and $\eta^{A}$ is the antenna polarization spinor. This form is required so that if any basis transformation is applied to the wave spinor, an inverse basis transformation will be applied to the antenna spinor such that the inner product is maintained invariant. Formally it is possible to express the wave spinor as the covariant derivative of the voltage with respect to the antenna spinor,

$$
\psi_{A}=\frac{\partial}{\partial \eta^{A}} V
$$

A full formal derivation of the antenna spinor from first principles would be out of the scope of the present paper, but we have shown in [41] that using Schelkunov's reaction theorem [31], [32] that the receive voltage can be expressed in terms of the reaction between the far field radiation of the antenna and the incoming plane wave in invariant tensor form that can be reduced to the spinor relationship (5). It is, however, possible to provide an intuitively obvious derivation of the height spinor which effectively mirrors that of the wave spinor, $\psi_{A}$ from the vector potential $\Phi_{A A^{\prime}}$. Here the usual construction of the spinor representation from the 4-vector combination of electric scalar potential and magnetic vector potential [21] is applied,

$$
\Phi_{a}=\left(\begin{array}{c}
\varphi \\
A_{x} \\
A_{y} \\
a_{z}
\end{array}\right) \rightarrow\left(\begin{array}{cc}
\varphi-A_{z} & -A_{x}+j A_{y} \\
-A_{x}-j A_{y} & \varphi+A_{z}
\end{array}\right)=\Phi_{A A^{\prime}}
$$

In [21], the objective in obtaining a pure 2-spinor representation was to 'lose' the primed index of the vector potential by contracting it (Penrose and Rindler [23] refer to the operation as 'transvection') with a fixed reference spinor, which we called the phase flag. Thus, formally by summation over the repeated index,

$$
\psi_{A}=\Phi_{A A^{\prime}} \bar{\theta}^{A^{\prime}} .
$$

In the standard coordinate frame, and circular polarization basis, this reads,

$$
\begin{aligned}
\left(\begin{array}{l}
\psi_{0} \\
\psi_{1}
\end{array}\right) & =\left(\begin{array}{cc}
0 & \Phi_{01^{\prime}} \\
\Phi_{10^{\prime}} & 0
\end{array}\right)\left(\begin{array}{l}
\bar{\theta}^{0^{\prime}} \\
\bar{\theta}^{1^{\prime}}
\end{array}\right)= \\
& =\left(\begin{array}{cc}
0 & \Phi_{01^{\prime}} \\
\Phi_{10^{\prime}} & 0
\end{array}\right)\left(\begin{array}{l}
1 \\
1
\end{array}\right)=\left(\begin{array}{l}
\Phi_{01^{\prime}} \\
\Phi_{10^{\prime}}
\end{array}\right)
\end{aligned}
$$

It will be recalled that the vector potential can be manipulated by gauge transformations [21], and the zeroes on the diagonal arise because the scalar potential is irrelevant to a propagating wave and because with the choice of the transverse gauge it has no longitudinal component. The remaining off-diagonal components $\Phi_{10^{\prime}}$ and $\Phi_{01^{\prime}}$ constitute, respectively, the left and right circularly polarized components of the wave.

The construction (9) may appear somewhat artificial, but the function of the components of $\bar{\theta}^{\prime}$ is to give (equal) amplitude weighting to the two independent circular polarization components of the vector potential, but also to fix their phases. There is no intrinsic angle in a plane circularly polarized wave which determines the zero phase point. This has to be established by convention, and the introduction of the phase flag is an explicit acknowledgement of the fact. We stress that the choice of the phases for the components of the phase flag is purely convention. The particular choice made here is not just the 
simplest numerically, but can be expressed in a geometrically invariant manner, by linking it to the spin-frame aligned with the propagation axis of the wave. It is recalled that a pair of normalised spinors making up a spin frame define a spatial reference frame unambiguously. Essentially, the two elements of the spin frame ordered pair define the null 4-vectors for two counter-propagating plane waves on an axis, while their relative phases determine the directions of a pair of orthogonal real axes in the plane of the wave.

For the spin-frame $\{\kappa, \lambda\}$ defined by the order normalised spinor pair, $\kappa^{A}$ and $\lambda^{A}$ such that

$$
\kappa_{A} \lambda^{A}=1
$$

and such that the longitudinal axis is aligned with the wavevector $k_{a}$, the first spinor of the pair maps to the wave-vector via the standard hermitian to 4-vector mapping,

$$
\kappa_{A} \bar{\kappa}_{A^{\prime}} \rightarrow k_{a}
$$

while, the other Hermitian combination, $\lambda_{A} \lambda_{A^{\prime}}$ determines the counter-propagating wave vector. For the standard coordinate reference frame (propagation along the $\mathrm{z}$-axis),

$$
\kappa^{A}=\left(\begin{array}{l}
1 \\
0
\end{array}\right), \quad \lambda^{A}=\left(\begin{array}{l}
0 \\
1
\end{array}\right) .
$$

Our convention is that the conjugate of the phase flag is given by

$$
\bar{\theta}^{A^{\prime}}=\bar{\kappa}^{A^{\prime}}+\bar{\lambda}^{\prime} A=\left(\begin{array}{l}
1 \\
1
\end{array}\right) .
$$

We now consider, analogously, how the antenna polarization spinor may be defined. It may be recalled that a 4-vector can be converted to a spinor representation as,

$$
x^{a}=\left(\begin{array}{l}
t \\
x \\
y \\
z
\end{array}\right) \rightarrow\left(\begin{array}{cc}
t+z & x-j y \\
x+j y & t-z
\end{array}\right)=X^{A A^{\prime}} .
$$

In the case of an antenna radiating in the $z$ direction, its effective height vector may be represented as,

$$
h^{a}=\left(\begin{array}{c}
0 \\
h_{x} \\
h_{y} \\
0
\end{array}\right) \quad \rightarrow \quad\left(\begin{array}{cc}
0 & h_{x}-j h_{y} \\
h_{x}+j h_{y} & 0
\end{array}\right)=H^{A A^{\prime}}
$$

Clearly, there is no timelike component to the antenna, and the dyadic Green's function projects out any longitudinal component of the dipoles that together combine to represent the entire antenna. This two-dimensional vector when restricted to the aperture plane is the contravariant equivalent to a Jones vector. We may consider complexified extensions to it, but strictly it is not transformed unitarily. Structurally $H^{A A^{\prime}}$ is similar in form and complementary to the spinor representation of the vector potential. It is an obvious step to reduce to spinor form by another phase flag $\tilde{\theta}_{A}$, so that, by transvection,

$$
\eta^{A}=H^{A A^{\prime}} \overline{\tilde{\theta}}_{A^{\prime}},
$$

where $\eta^{A}$ is the antenna height spinor (which does transform properly under a unitary $\mathrm{SU}(2)$ representation) which like the wave spinor is represented geometrically by a generator of the Poincaré sphere. The spinor, $\overline{\tilde{\theta}}_{A}^{\prime}$ is now the antenna phase flag (which we now must distinguish from the wave phase flag). Again the function of this phase flag is to weight equally the amplitudes of the independent circular polarization components of the radiated field, and to set their phases. One can conceive this in a concrete way by considering an antenna with two circular polarization ports: the overall phase of the phase flag sets the antenna phase centre, while the relative phases of the components can be related to a number of factors, such as the relative orientation of the ports, and the lengths of transmission line from the point where the phases are gauged. Conceivably, there may be bistatic observation schemes in which either or both antennas may be steered through a sequence of pointing directions. When coherent phase is important, care need to be exercised in such operations, because topological phase, otherwise known as Berry's phase [42], which is well known in optics, can be introduced. This is a phenomenon known as a holonomy, in which if the antenna's pointing direction is taken around a finite loop with its reference direction parallel to the path, the phases at the end do not match the starting phases. It follows that the polarization phase changes are sensitive to the path followed from one direction to another. By expressing a continuous sequence of rotations in the unitary $\mathrm{SU}(2)$ representation the phase shifts may be computed by applying the unitary matrices to the phase flag.

So far it has not been indicated what relation might exist between the wave and antenna phase flags. For there to be an invariant voltage equation (5), there must be a relation that ties it to the tensor representations of the vector potential and antenna height vector, $h^{a}$. It is therefore found that the condition

$$
\theta^{0} \tilde{\theta}_{0}=\theta^{1} \tilde{\theta}_{1}
$$

must apply in the standard basis and frame. It is then found that,

$$
\overline{\tilde{\theta}}_{A^{\prime}}=\left(\begin{array}{l}
1 \\
1
\end{array}\right) .
$$

That $\overline{\tilde{\theta}}_{A^{\prime}}$ is not simply $\varepsilon_{A^{\prime} B^{\prime}} \bar{\theta}^{B^{\prime}}$ can be explained by the fact that the wave vector received by the antenna is antiparallel to that which it transmits (that which is involved in the Schelkunoff reaction). The antenna phase flag is actually the covariant form of the phase flag associated with the wave it transmits. This is derived from the spin-frame for the outgoing wave, which must be expressed as $\{\lambda,-\kappa\}$ because the spin frame inner product must normalise as +1 ; flipping the order of a spinor inner product reverses the sign, because of its skew metric $\varepsilon_{A B}$. On lowering the index of $\tilde{\theta}_{A}$, the negative sign is removed.

For the wave travelling in the $z$ direction, this means that each circularly polarized component of the wave is deemed to have $0^{\circ}$ or $180^{\circ}$ phase in the $x y$ plane when the field-vector crosses the $x z$ plane.

\section{SCATTERING EXPRESSED IN SPINOR FORM}

From the previous arguments, it follows that the general bistatic scattering relationship can be expressed in spinor form 
as the voltage equation

$$
V=\frac{I Z_{0}}{\lambda r_{1} r_{2}} \eta^{A} S_{A B} \xi^{B}
$$

in which $\eta_{A}$ and $\xi^{B}$ are the transmit and receive antenna spinors respectively, and $S_{A B}$ is the bilinear form usually known as the scattering matrix. In the sequel, the scalar factor depending on the drive current $I$, ranges $r_{1}$ and $r_{2}$, the wavelength $\lambda$, and impedance of free space $Z_{0}$ will be considered absorbed into the scattering matrix, assuming unit drive current. The four components of $S_{A B}$ express the response for each co-polar and cross-polar combinations in the respective bases of the antennas. Fundamentally, this is what is measured, while the abstracted version of the scattering matrix, which relates the incident and scattered fields is deduced. In practical terms, (19) can be conceptualised initially in terms of the parametric representations of the polarization states. In other words the polarization spinors can be considered in terms of the polarization ratios and their amplitudes. What is not immediately apparent is that $S_{A B}$ can be referenced to a single coordinate system. This is because it was established in [21] that the spinor representation of the wave essentially holds in any spatial reference frame - this being down to the double covering of the rotation group $\mathrm{SO}(3)$ by the unitary group $\mathrm{SU}(2)$. Consequently, we can infer geometric properties associated with the scattering operator $S_{A B}$. Just as the geometric properties of an inertia tensor capture its invariant descriptors irrespective of the spatial frame in which the tensor is evaluated, knowledge of the existence of invariant properties of a scattering operator leads to methods to obtain such invariants regardless of the polarization basis. To date, this has been almost impossible to establish for bistatic scattering because the ability to choose polarization bases independently for each antenna makes the problem so loose that the only invariants obtainable are the absolute singular values and determinant. To determine the intrinsic geometric properties of a scattering operator, it becomes necessary to return to spatial coordinates. In the next section, we show how a geometric relation is determined by considering the projective geometric representation of the antenna dual of the Jones vector.

\section{GeOMETRIC CORRESPONDENCE FOR BISTATIC SCATTERING}

\section{A. Motivation for the geometric model of bistatic scattering}

To motivate the argument, it is best first to consider initially only linear polarizations. We consider an arbitrary scattering geometry. Supposing the two antennas in the scattering experiment were equivalent to linear dipoles that can be rotated to realise all possible polarizations in transmit and receive. If one were to imagine a laser pointer aligned with each dipole, such that it could 'project' onto the sky, the complete set of polarizations for each antenna would describe an arc on the celestial sphere. Making each laser point both ways allows for a direct representation of the projective plane, so that we deal with lines rather than arcs, as shown in Fig. 1 and Fig. 2.

If instead we consider also elliptical and circular polarizations for a given antenna orientation the points on the

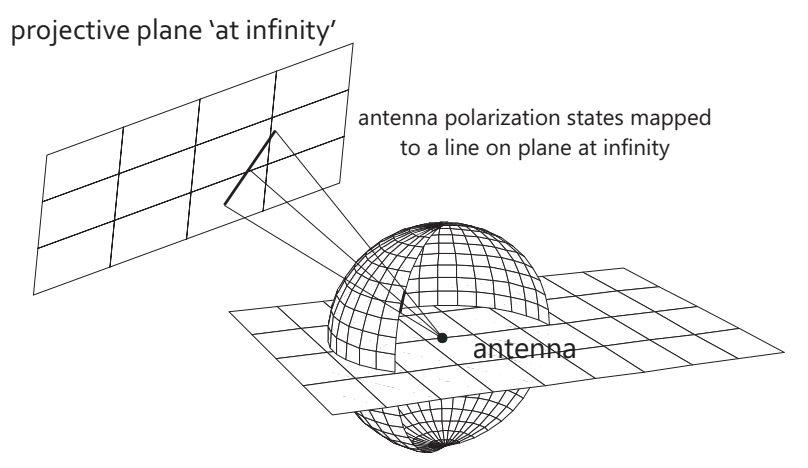

Fig. 1. Prolongation of the antenna dipole axes of an antenna to the celestial sphere, or plane at infinity maps the dipole state to a point on a line of the plane. An antenna has its own line determined by the intersection of its aperture plane with the plane at infinity.

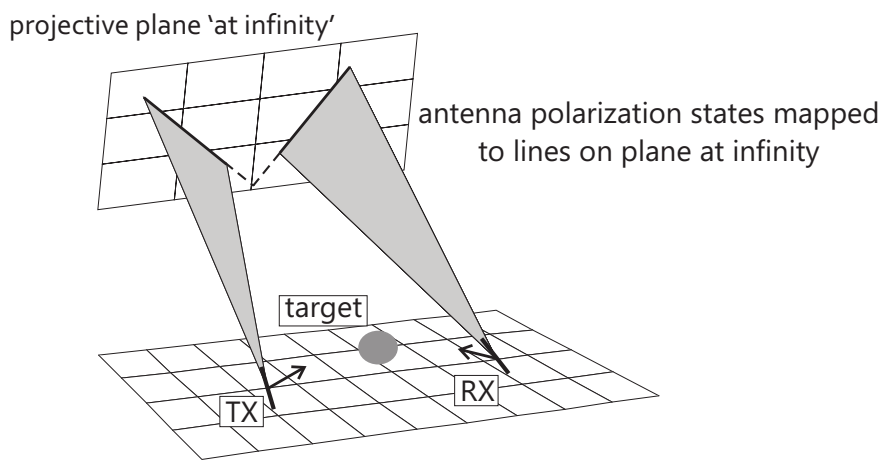

Fig. 2. Transmit and receive antennas: a scatterer determines a unique reciprocal mapping between the lines corresponding to transmit and receive antennas.

celestial sphere and consequently on the projective plane become complex. We cannot easily visualise complex points on a line but the important point is that the complex analytic geometric framework works (the algebraic approach means it has to!) in exactly the same way for complex polarization ratios as for the real ones (linear polarizations).

It can be understood that for each transmit state there must be a receive state that nulls or minimises the return power. For example, a horizontally polarized antenna, corresponds (or, for emphasis, is in null-correspondence) with a vertical polarized wave. This concept may be generalised so that if there is a null return from a scatterer, the transmit and receive states may then be said to correspond with respect to the scatterer. The concept of correspondence is expressed rigorously in [41] expressing the polarization states (wave and antenna) in spinor form. Polarimetry already encompasses the idea of orthogonal states being antipodal on the Poincaré sphere. In projective geometry the idea of correspondences is common, and often significant geometric relationships are most clearly expressed in this way. In this work, the concept of correspondence in polarimetry is the fundamental physical phenomenon that allows us to represent the scattering process as a geometric conic which maps polarization states. The linear nature of scattering means that there is a geometric relationship established by this correspondence. By extending to complex polarizations, generalising naturally to complex coordinates 
along each line of representation for the antenna states, it is then guaranteed that there is a unique null receive state. The linear nature of scattering matrices ensures that the geometric relationship is of a well determined form, and it is in fact a consequence of a theorem of the 19th century geometer, Steiner [24] that such a relationship determines a unique conic curve (such as an ellipse) in the plane. As will be seen, the scattering geometry in part determines the conic, while the properties of the scattering matrix are related to a projective invariant of the conic.

\section{B. Polarity relations}

As has been emphasized before in outlining the principles of Geometric Polarimetry, the Poincaré sphere is the invariant object under all linear transformations that are admissible [21]. The key point is that we refer polarization states before and after scattering to the same Poincaré sphere. Since the Poincaré sphere is analogous to the metric tensor of special relativity theory (through its standard coordinate representation) it plays a central role in relating geometric objects of complementary rank as the metric tensor does in relating contravariant and covariant tensors. The geometric relation defined by a quadric surface that is analogous to index raising or lowering is that of polarity. In general terms, in 3D projective geometry, a polarity with respect to any quadric surface provides a one to one correspondence between points and planes, which are analogues of contravariant and covariant 4 -vectors. A given plane intersects a quadric in a plane conic curve, and the tangent planes to the quadric at all points of that curve intersect in a unique point, said to be polar to the plane with respect the quadric. The relation of polarity is evidently a symmetric one. By extension, a polarity also determines one to one correspondences between pairs of lines. Two lines are polar if the tangent planes at the points where one line intersects the quadric intersect in the other line. Furthermore, the polar planes for every point on the first line all pass through the second line. In trying to visualize such cases it is important to bear in mind that quadric surfaces are all equivalent in complex projective geometry, so that intersections are generic; for example, any line intersects any quadric generally twice (in real or complex points), although the points may coincide. The only exception to this rule is when the line in question is a generator of the quadric, in which case all points are in the quadric surface. Generators are said to be self polar. A simple example of working with complex geometry would be to consider intersections of the line $y=2$ with the circle $x^{2}+y^{2}=1$ in the plane. In real geometry there are no intersections, while in the complexified plane there are two: $( \pm i \sqrt{3}, 2)$. This is more than a curiosity, because, if we constructed the complex tangent lines at those intersection points, they would be found to intersect at $\left(0, \frac{1}{2}\right)$ which is the point that is polar to the line. That is, algebraically we can carry out the same process of construction as would be the case with a line that intersected the circle in real points to find its polar point.

\section{The plane of antenna vectors}

As noted in section IV, antenna vectors lie in a two dimensional subspace of the projective polarimetric space. These are dual to the Jones vector states discussed in [25]. This condition arises because the operation of projecting out time-like components of the current elements which represent the source of the field in (as shown in [41]) means that they lie in a definite plane $\Pi_{a}$ defined in coordinate representation by

$$
\Pi_{a}=g_{a b} t^{b}=\left(\begin{array}{rrrr}
1 & 0 & 0 & 0 \\
0 & -1 & 0 & 0 \\
0 & 0 & -1 & 0 \\
0 & 0 & 0 & -1
\end{array}\right)\left(\begin{array}{l}
1 \\
0 \\
0 \\
0
\end{array}\right) .
$$

To be clear, all the antenna height vectors for arbitrary direction of wave vector are confined to this coordinate plane. Geometrically, this plane is polar to the centre of the Poincare sphere. Points lying in the plane $\Pi_{a}$ are characterised by the relation

$$
\left(\begin{array}{l}
1 \\
0 \\
0 \\
0
\end{array}\right) \cdot\left(\begin{array}{l}
0 \\
x \\
y \\
z
\end{array}\right)=0
$$

For arbitrary $x, y, z$, the inhomogeneous coordinates $\left(\frac{x}{0}, \frac{y}{0}, \frac{z}{0}\right)$ are infinite. In an affine space, this is referred to as the plane at infinity.

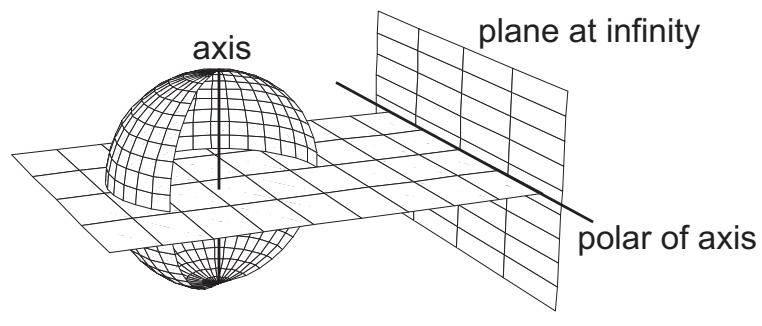

Fig. 3. The relationship between an axis of the sphere and its polar line on the plane at infinity.

It is the great advantage of a projective model, using homogeneous coordinates, that representing such points presents no problem. Considering further what constraints apply to points representing antenna height vectors for a fixed pointing direction, it becomes obvious that such points must belong to a fixed line in $\Pi_{a}$. Geometrically, the line in question must be the polar to the axis of the spin frame of the antenna as shown in Fig. 3. What this means is that all the polarization states of the antenna are uniquely represented on this line as briefly introduced in [43]. In the case of real linear polarizations, the line is simply the collection of the projections of directions of all polarization vectors on the celestial sphere. For more general polarization states, one has to consider not just real points on the line but complex points. These can be obtained parametrically by weighting two real base points by complex weights, and the ratio of the weights is synonymous with the concept of polarization ratio if the base points are suitably chosen. 
The representation we are building up here immediately leads to a convenient geometric model for bistatic scattering by considering the two lines representing the respective pointing directions for the antennas associated with the incident and scattered waves. Then, the scattering measurements that can be made for these two directions imply the existence of a geometric homographic correspondence between the two lines. Specifically, a homography exists if the projective parameters of the two lines (see [21]), $\rho_{1}$ and $\rho_{2}$ are connected by a relation of the form

$$
\left(\begin{array}{c}
\rho_{2} \\
1
\end{array}\right)\left(\begin{array}{ll}
a & b \\
c & d
\end{array}\right)\left(\begin{array}{c}
\rho_{1} \\
1
\end{array}\right)=0
$$

The relationship between polarization ratios and scattering matrix clearly meets this definition. From this point of view, the concept of corresponding antenna states for which the received voltage is null (as introduced in [41]) ties in perfectly with this model, illustrated schematically in Fig. 4. The asymmetry of bistatic scattering is represented in the model by the fact that a homographic correspondence between lines in a plane is considered to be a directional mapping from one to the other. For, if the ordering is swapped in (22) the matrix is transposed. The bi-linearity of the relationship is sufficient to establish

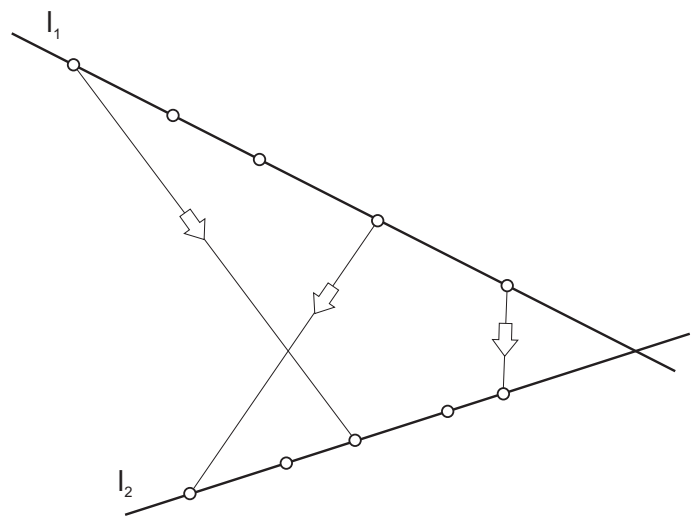

Fig. 4. Schematic representation of a homographic correspondence of two lines in a plane. Here the points of the lines $l_{1}$ and $l_{2}$ represent all possible polarization states of the two antennas, and the correspondence represents the cases for which the receive antenna is isolated from the transmitter via the scattering operation.

the validity of the homographic model [24]. What follows from this argument is that we can draw on known theory in projective geometry to determine some important invariant properties that have not previously emerged in the treatment of this topic. What makes this a useful representation is that the correspondence has a highly structured geometric description, in the sense that (via the dual form of a theorem of Steiner [24]), the lines joining corresponding states are all tangent to a fixed conic (such as an ellipse, etc..) as depicted schematically in Fig. 5. The states $P, Q$ and $P^{\prime}, Q^{\prime}$ are correspondent.

More detailed constructions are depicted in Fig. 6. The line $F F^{\prime}$ is the cross-axis of the homography, such that for any pair of correspondences, $P_{1} P_{1}^{\prime}$ and $P_{2} P_{2}^{\prime}$ the lines $P_{1} P_{2}^{\prime}$ and $P_{2} P_{1}^{\prime}$ intersect on it. The conic is tangent to the lines $P Q$ for the transmit antenna and $P^{\prime} Q^{\prime}$ for the receive antenna at $F$ and $F^{\prime}$ respectively, so that $O$ is the state on $P^{\prime} Q^{\prime}$ corresponding

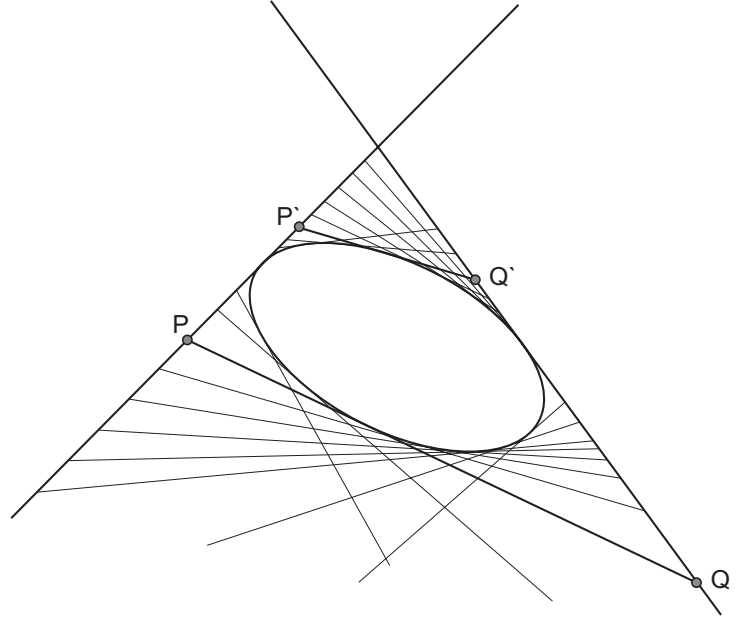

Fig. 5. The dual form of a theorem of Steiner: the lines joining corresponding states are all tangent to a fixed conic.

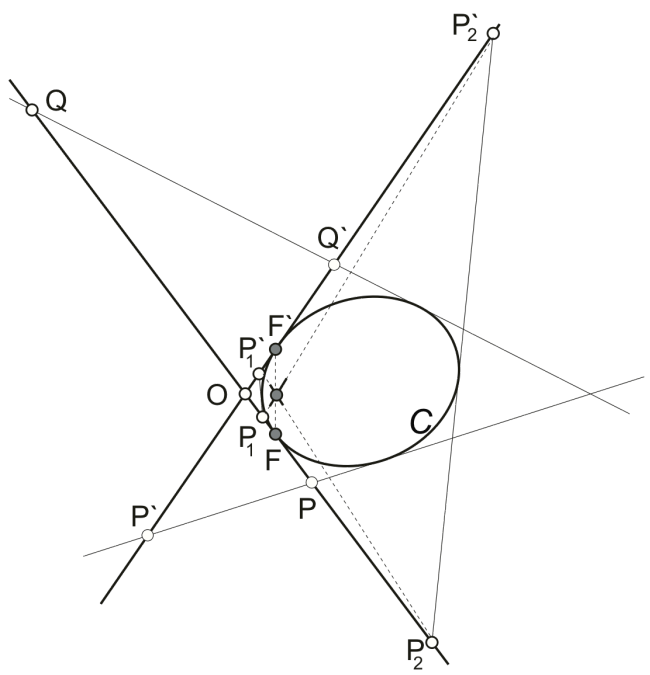

Fig. 6. Construction of the cross axis of a homography.

to $F$ and $F^{\prime}$ is the state on $P^{\prime} Q^{\prime}$ corresponding to $O$ on $P Q$. Given that the conic $C$ is tangent to these lines at fixed points, it is uniquely determined by a single complex constant $k$, such that if $O F F^{\prime}$ are taken as coordinate base points $X_{0}, X_{1}, X_{2}$, the conic has a canonical representation in plane homogeneous coordinates as

$$
\left(\begin{array}{l}
x_{0} \\
x_{1} \\
x_{2}
\end{array}\right)\left(\begin{array}{rrr}
0 & 0 & 1 \\
0 & -k & 0 \\
1 & 0 & 0
\end{array}\right)\left(\begin{array}{l}
x_{0} \\
x_{1} \\
x_{2}
\end{array}\right)=0 .
$$

If $O$ is chosen as unit point on $P Q$ and on $P^{\prime} Q^{\prime}$ as base points then the parameterizations of $F$ and $F^{\prime}$ are $k, \frac{1}{k}$, with those 
of $M$ and $N$ being 0 and $\infty$ respectively. This arrangement is consistent with the canonical representation [24] of the homography as

$$
\left(\begin{array}{l}
1 \\
t
\end{array}\right)\left(\begin{array}{rr}
0 & 1 \\
-k & 0
\end{array}\right)\left(\begin{array}{c}
1 \\
t^{\prime}
\end{array}\right)=0
$$

or, $t^{\prime}=k t$, where $t$ and $t^{\prime}$ are, respectively parameters with respect to base points $P Q$ or $P^{\prime} Q^{\prime}$, such that for a point $X$ on the line $P Q$,

$$
\begin{aligned}
& X(t)=P+Q t \\
& X(1)=O
\end{aligned}
$$

The complex parameters $t$ and $t^{\prime}$ are equivalent to polarization ratios so (24) is a canonical representation of the voltage equation (1) for corresponding states.

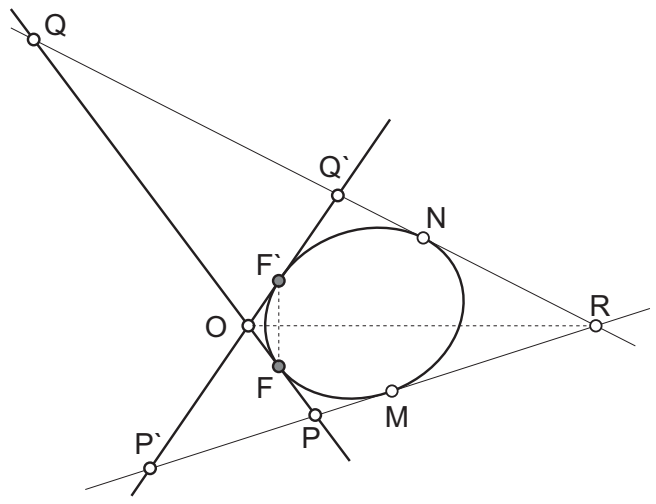

Fig. 7. Schematic geometry of the homographic correspondence involving the invariant conic. Tangents to the conic join the states on $P Q$ to their corresponding states on $P^{\prime} Q^{\prime}$. The line $F F^{\prime}$ is the cross axis of the homography.

What is important about this is that $k$ is said to be a projective invariant of the homography. By implication, this projective invariant characterises the bistatic matrix associated with the homographic mapping. This is a completely new result in bistatic scattering, which it seems could hardly have been discovered except by this geometric model. What remains to be done is to relate this result to the scattering matrix. The way to proceed is to understand that the points in Fig. 7 are determined by the geometry and the scatterer together, while the canonical forms are obtained by, in general, projective, non-unitary transformations. But for each antenna, base points for a suitable unitary basis may be established independently. Reduction to a general scattering matrix is therefore a question of determining the coordinates in the respective bases of $O, P$, $Q, F$ and $O, P^{\prime}, Q^{\prime}, F^{\prime}$. In this scenario, the degree of freedom accorded by independent choices of basis for the respective antennas is so broad that it would be difficult to interpret results. Also, an extra layer of complexity is introduced by the fact that the polarization states of the antennas would have to be considered as belonging to different spin states. This further entails a notational distinction for the indices of the respective spinors, such as in [25] use of $A, \tilde{A}$, etc. Given the fundamental asymmetry of bistatic scattering, there is a simple solution which dramatically simplifies matters, allowing to reduce the scattering matrix to a relation expressed in a single spin frame. The key to this move is to observe that a perspective mapping from $P^{\prime} Q^{\prime}$ back to $P Q$ from $R$ as a centre allows to compound the homography of the scattering process from $P Q$ to $P^{\prime} Q^{\prime}$ with a mapping in the reverse direction. In this case the compound homography is reduced to a homography from a line to itself. The mapping (16) that reduces an antenna state to a spinor is an elementary homography that effectively converts inhomogeneous polarization ratios to homogeneous spinors with the identical polarization ratio. In observing that the reverse homography, centred on $R$ is effectively an identity operation - $P O Q, P^{\prime} O Q^{\prime}$ respectively map to $0,1, \infty$ - the projective invariant $k$ is unchanged when the scattering matrix is represented as a covariant spinor form in the frame of the transmit antenna. In this representation, the determination of the characteristic polarizations is extremely straightforward. The pairs $P P^{\prime}$ and $Q Q^{\prime}$ appear in this representation as linked to $M N$ as 'united points' of the homography, and analogous to Huynen's copolar nulls. Indeed, they are found via the same characteristic equation (24). Because the projective invariant $k$ is valid for the spinor form of the scattering matrix, it is clear that $k$ is the ratio of characteristic values. The combination of united polarizations and characteristic values is sufficient to reconstruct all the characteristic polarizations $P, F, Q$ and $P^{\prime}, F^{\prime}, Q^{\prime}$ for the respective antenna states.

\section{VISUALIZATION OF EXAMPLES OF BISTATIC SCATTERING WITH REAL GEOMETRY}

There are two important special examples in which the geometric construction for the bistatic scattering involves only real geometry, and can therefore be fully visualized. These are the electrically small sphere and dipole (Rayleigh scattering cases).

\section{A. Small spheres}

The case of small spheres is simpler in that there are no free parameters in the problem other than the bistatic scattering angle. To illustrate this case we adopt a coordinate system where the $x y$ plane is the scattering plane containing the incident and scattered wave vectors. Taking the origin of the coordinate system at the target, we consider the respective polarization planes, parallel to the aperture planes of the antennas, that pass through the scatterer, intersecting in the $z$ axis, at a bistatic angle of $\alpha$ (zero being the monostatic case). Without loss of generality the transmit wave is considered to be propagating along the $y$ axis. For linear polarization at an angle of $\theta_{t}$ to the $z$ axis, the transmitted electric field is parallel to the antenna height vector $\mathbf{h}_{t}$,

$$
\mathbf{h}_{t}=\left(\begin{array}{c}
\cos \theta_{t} \\
0 \\
\sin \theta_{t}
\end{array}\right) \text {. }
$$

Fig. 8 illustrates this together with the plane through the origin orthogonal to this vector, (its polar), $\pi\left(\mathbf{h}_{t}\right)$. It is seen that the linear polarization vector is uniquely represented by a point on any plane of constant $z$. Because only ratios are involved, it is convenient to take the plane at infinity. Although this introduces some awkwardness in Euclidean geometry, it 
becomes a natural assumption in a projective representation in which infinities are not exceptional.

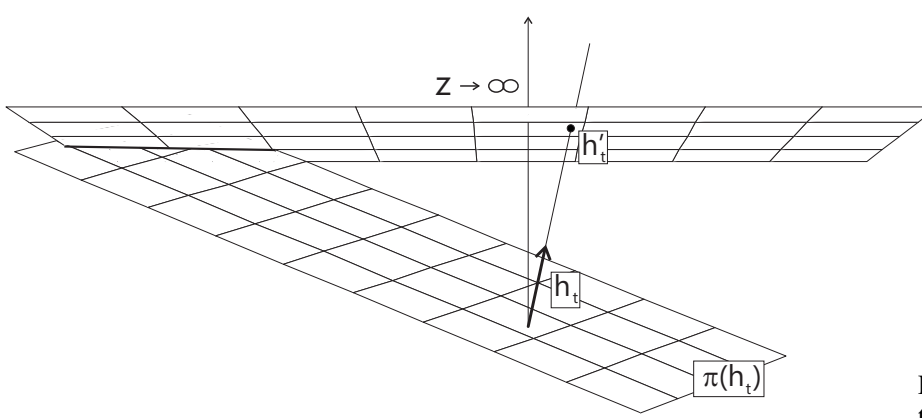

Fig. 8. The transmit polarization plane. Associated with the antenna height vector $\mathbf{h}_{t}$ is a point $\mathbf{h}_{t}^{\prime}$ in the plane of constant $z$ as $z$ tends to infinity. The plane, $\pi\left(\mathbf{h}_{t}\right)$, polar to $\mathbf{h}_{t}$ is the equatorial plane relative to $\mathbf{h}_{t}$ as axis.

For the receive antenna, the resolving vector $\mathbf{h}_{r}$ projected back along the receive wave vector $\mathbf{k}$ to the scatterer at the origin is

$$
\mathbf{h}_{r}=\left(\begin{array}{c}
\cos \alpha \cos \theta_{r} \\
\sin \alpha \cos \theta_{r} \\
\sin \theta_{r}
\end{array}\right),
$$

where $\alpha$ is the polar angle and $\theta_{r}$ the azimuth angle of the receiving antenna height vector. We now extend the vectors to their intersection with the plane $z=\infty$, as shown in Fig. 9. The points at which the extended height vectors meet the

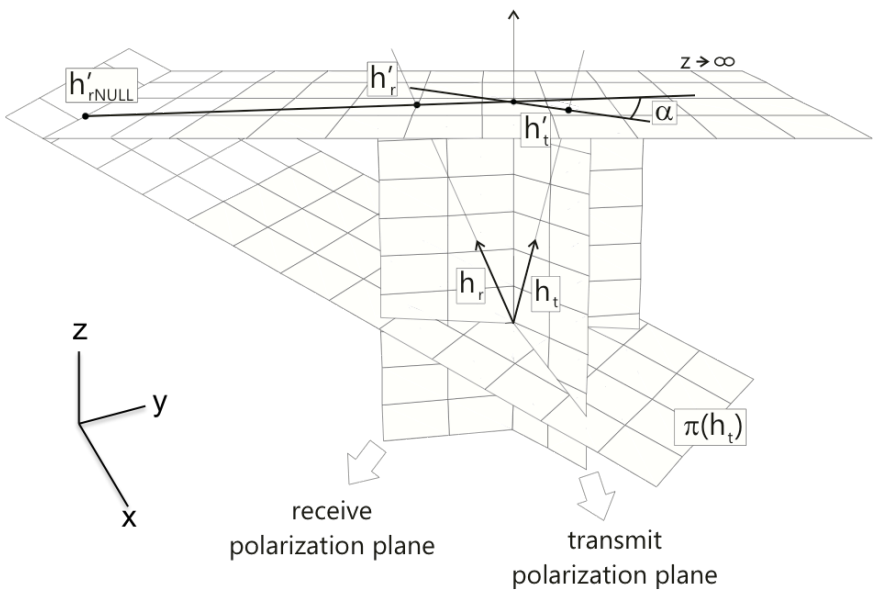

Fig. 9. Construction of the receiver null antenna state $\mathbf{h}_{r \text { NULL }}$ for bistatic sphere. The receive antenna state for a null is found at the intersection of the receive polarization plane and the plane $\pi\left(\mathbf{h}_{t}\right)$, polar to $\mathbf{h}_{t}$.

plane at infinity have distances from the $z$-axis in the ratios of

$$
\cot \theta_{t}: \cot \theta_{r}=\rho_{t}: \rho_{r},
$$

where $\rho_{t}$ and $\rho_{r}$ are the respective linear polarization ratios in each plane as shown in Fig. 10.

Since for the sphere the dipole induced is parallel in (3D) space to the incident field, the required condition for the received voltage to be null is that the receive antenna polarization should lie in the intersection of the planes polar to $\mathbf{h}_{t}$ and $\mathbf{k}$ (the direction $\mathbf{h}_{t} \times \mathbf{k}$ ). It may at first sight appear that applying the criterion that the receive antenna be orthogonal

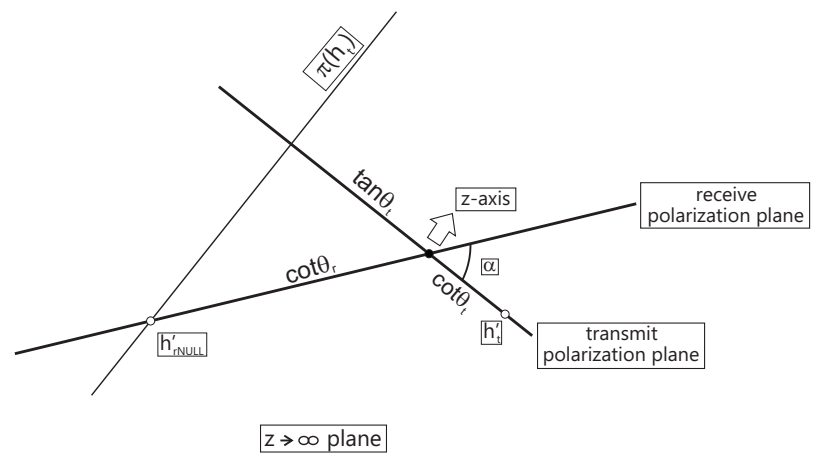

Fig. 10. Construction of the receiver null antenna state $\mathbf{h}_{r \text { NULL }}$. Looking down the $z$-axis onto a plane of constant $z$, the transmit and receive polarization planes intersect it in lines on which the respective antenna polarization states are represented as points by prolongation of their antenna height vectors.

to the radiating dipole itself - without accounting for the geometrical effect of projecting forward only the part parallel to the receive polarization plane - may be naively incorrect; however, the projection operation arises from the repeated cross product $\left(\mathbf{h}_{t} \times \mathbf{k}\right) \times \mathbf{k}$. To find the null receive state the second cross product is not needed, because it produces the received field state which is orthogonal to the antenna state required to null. The resulting parametric equation for null correspondence between the transmit and receive antennas involving the polarization ratios in the respective polarization planes is therefore,

$$
\rho_{t} \rho_{r} \cos \alpha+1=0,
$$

which may equivalently be expressed in terms of the voltage form of the scattering matrix as,

$$
\left(\begin{array}{c}
\rho_{t} \\
1
\end{array}\right)\left(\begin{array}{cc}
\cos \alpha & 0 \\
0 & 1
\end{array}\right)\left(\begin{array}{c}
\rho_{r} \\
1
\end{array}\right)=0
$$

In this case the scattering matrix is seen to be symmetric for all bistatic angles - a consequence of the high symmetry of the scatterer itself. The case $\alpha=0$ is the usual monostatic relation while for $\alpha=\frac{\pi}{2}$ the result corresponds to the famous explanation in terms of Rayleigh scatter for the polarization of scattered sunlight in the sky at $90^{\circ}$.

The lines joining the null-corresponding states form the tangential envelope of a real hyperbola asymptotic to the polarization planes. Fig. 11 shows illustrates the envelopes for bistatic angles $30^{\circ}$ degrees and $60^{\circ}$.

\section{B. The elementary case of a dipole}

In the elementary case of a dipole $\mathbf{p}$, the dipole excitation by an incoming wave is parallel to the dipole, and vanishes if the incoming polarization $\mathbf{h}_{t}$ is in the orthogonal plane $\pi$. Likewise, if the receive antenna $\mathbf{h}_{r}$ is in the same plane there is no received voltage. Diagrammatically, this is represented in Fig. 12.

The scattering plane is $z=0$. The antenna states are represented in the plane $z=\infty$ by the prolongation of their polarization vectors. In this case, the conic representing the dipole scattered is in this case clearly degenerate and comprises the intersection of $\pi$ and $z$ with $\mathbf{h}_{t}$ and $\mathbf{h}_{r}$ respectively. 


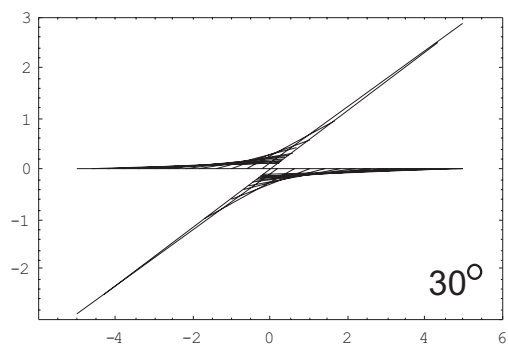

(a)

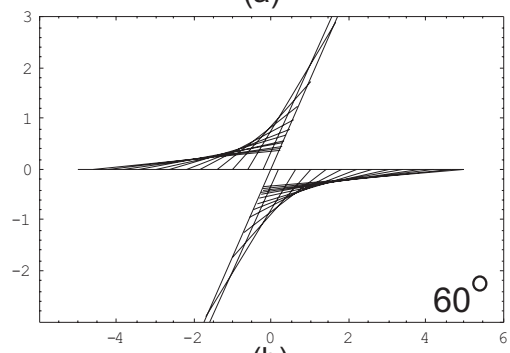

(b)

Fig. 11. The lines joining null-corresponding antenna states in the $z$-plane representation on the plane at infinity for scattering from a Rayleigh sphere envelop real hyperbolae. Examples illustrated are for bistatic angles of (a) $30^{\circ}$, (b) $30^{\circ}$.

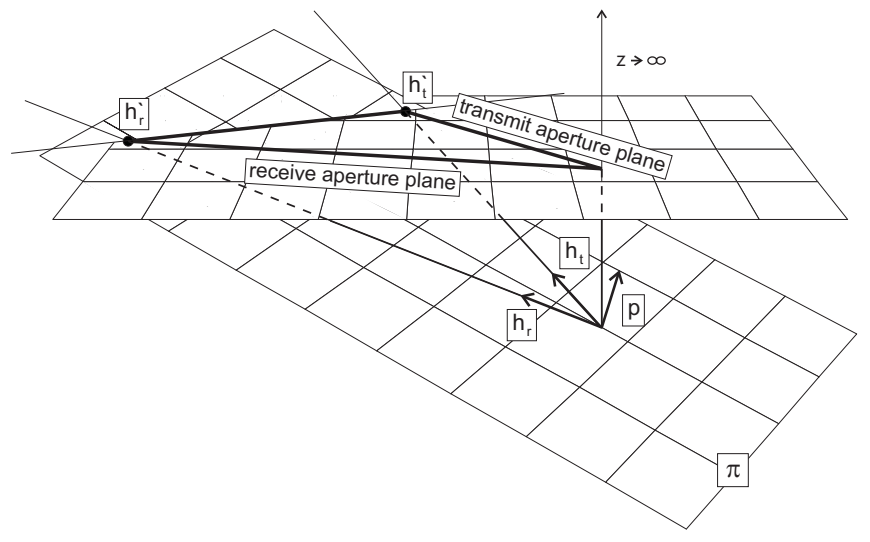

Fig. 12. The construction of conic for the dipole $\mathbf{p}$ for incoming polarization and receive antenna in the plane $\pi$ orthogonal to $\mathbf{p}$.

Conversely, for a small Rayleigh sphere the induced dipole is always parallel to the transmit antenna height vector, and the scattered field is the component of this projected into the receive aperture plane.

\section{Discussion}

The examples discussed show a pure geometric construction in physical space which carries all the information about the scattering matrix except its magnitude. Specifically, this representation exists independently of any choice of polarization basis. Although the sphere and dipole are special cases, the basic concept of the scattering matrix being characterised by an invariant conic on the plane at infinity remains true in general if the geometry of space is complexified, as we now demonstrate, using the more powerful projective framework. In part the geometry of the conic is determined by the scattering geometry, in that the property of being asymptotic (or tangent) to the polarization planes turns out to be general. As will be shown, there is also however in the general case a degree of freedom represented by a complex invariant, which can therefore be associated with the scattering matrix.

\section{THE BISTATIC HUYNEN POLARIZATION FORK}

One of the best known invariant descriptions of a scattering matrix is the Huynen Polarization fork [13]. In the case of monostatic scatterers, it identifies four characteristic polarizations, namely two copolar nulls and the transmit polarizations of minimum and maximum total power return are distributed symmetrically on a circle. The power extrema are orthogonal and their common axis bisects the copolar nulls (Fig. 13). Strictly speaking, the problem of determining power extrema

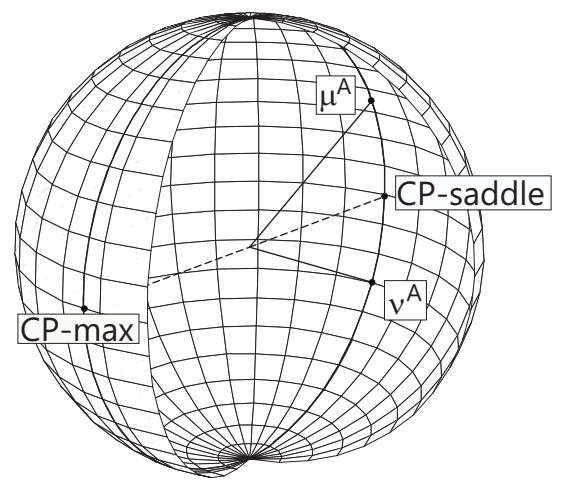

Fig. 13. Copolar nulls, copolar power return maximum and saddle point in the monostatic case.

is related to the incoherent form of the scattering matrix, and is not analytic in the mathematical sense. There are various approaches to determining the location of these extrema which amount in the end to the same thing. Whilst an SVD approach [19] leads to eigenvalues of the product of the scattering matrix with its conjugate, an instructive approach using the spinor algebra can be taken by constructing the matrix

$$
G_{B B^{\prime}}=S_{A B} \bar{S}_{A^{\prime} B^{\prime}} g_{0}^{A A^{\prime}} \text {. }
$$

The term $g_{0}^{A A^{\prime}}$ is the familiar g-spin tensor ( [23]) corresponding to the trace operator that acts on a coherency matrix. The left hand side of (31) is easily seen to be Hermitian symmetric, and bears the same relation to a Stokes vector as the coherency matrix does. $G_{B B^{\prime}}$ is Graves's matrix and its transvected form the Graves vector [29]. The extra index in (31) shows the Graves vector is actually the first row of the Kennaugh matrix, which resolves the received total power. The received power is therefore expressed in terms of the transmit antenna state $\eta^{A}$ as,

$$
P_{\text {тот }}=G_{A A^{\prime}} \eta^{A} \bar{\eta}^{A^{\prime}}
$$

which is the same as the inner product of the Graves' vector and the transmit Stokes vector. It is clear without further analysis that the extrema occur when the vectorial parts of the Stokes vector and Graves' vector are aligned parallel or antiparallel. Thus the power extrema are shown to be orthogonal on the Poincaré sphere. This result therefore holds equally for bistatic scatterers as for monostatic. When the scattering path 
is transposed, both the scattering matrix and the Kennaugh matrix are transposed. Owing to the asymmetry of the bistatic descriptors, it follows that a different Graves' vector arises for this case. There are therefore two Huynen forks associated with a bistatic scatterer according to the assignment of transmit and receive functions for the antennas.

One can consider the difference between the two cases as being attributable to the antisymmetric part of the resulting Kennaugh matrix which has the form of a real bivector (analogous to the Maxwell electromagnetic tensor (see [21])) and which can be expressed in spinor form, $N_{A B A^{\prime} B^{\prime}}$ as

$$
N_{A B A^{\prime} B^{\prime}}=\gamma S_{A B} \varepsilon_{A^{\prime} B^{\prime}}+\bar{\gamma} \bar{S}_{A^{\prime} B^{\prime}} \varepsilon_{A B}
$$

where the asymmetry factor is

$$
\gamma=\frac{1+k}{1-k}
$$

When $\gamma$ is real or imaginary, the vector part of $N$ contributing to the antisymmetric offset of the Graves' vector is either in the plane of the copolar nulls or orthogonal to it respectively. This means that when $\gamma$ is real the axis of the Huynen fork is skewed from symmetric in the plane of the copolar nulls; when imaginary, the skewing occurs in the orthogonal direction.

\section{A. An alternative unique Huynen fork}

In considering the standard definition of the Huynen fork, its axis was determined according to the copolar power return maximum and saddle point. Subsequently, it was determined that these polarizations are orthogonal on the Poincare sphere, but it turns out also that these are corresponding states for the scatterer. An alternative approach is to consider whether a unique pair of orthogonal corresponding states exists in the bistatic case. By geometric reasoning, it can be shown that the complex invariant $k$ for the scattering matrix must be related to the cross-ratio of any pair of corresponding states together with the copolar nulls. If we label the copolar nulls $\mu^{A}$ and $\nu^{A}$, while a pair of corresponding states are designated $\chi^{A}$ and $\rho^{A}$, then we have the projectively invariant cross ratio,

$$
k=\frac{\mu_{A} \chi^{A} \nu_{B} \rho^{B}}{\mu_{C} \rho^{C} \nu_{D} \chi^{D}} .
$$

Now, instead of requiring that the corresponding states are power extrema the alternative requirement may be stipulated that they are polarimetrically orthogonal. The requirement for such a pair of states introduces a constraint relating to unitarity that means the problem lacks analyticity in the spinors expressed algebraically. The best way to deal with this is to express the pair of orthogonal unit spinors in a trigonometric form as:

$$
\chi^{A}=\left(\begin{array}{c}
-\cos \frac{\psi}{2} \\
\sin \frac{\psi}{2} \mathrm{e}^{j \varphi}
\end{array}\right), \quad \rho^{A}=\left(\begin{array}{c}
\sin \frac{\psi}{2} \mathrm{e}^{j \varphi} \\
\cos \frac{\psi}{2}
\end{array}\right) .
$$

This prescription meets the spin frame normalisation $\chi_{A} \rho^{A}=$ 1 . Without loss of generality we take the trigonometric angles $\psi$ and $\varphi$ as spherical polar coordinates and assume the scattering matrix is transformed so that the copolar nulls are symmetrical disposed about the polar axis, and in the plane of the prime meridian $\varphi=0$, as shown in Fig. 14. Then,

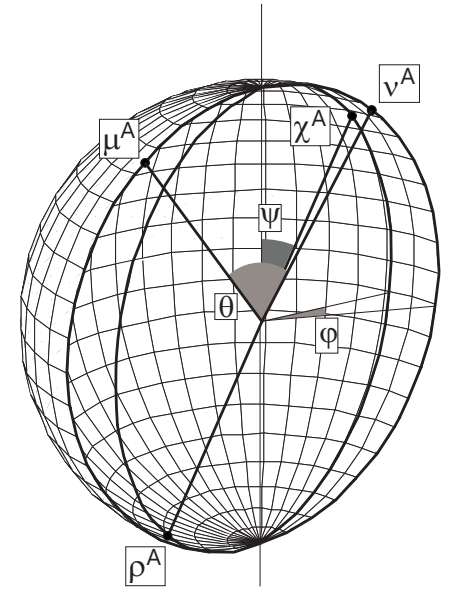

Fig. 14. Copolar nulls, copolar power return maximum and saddle point in the bistatic case.

$$
\mu_{A}=\left(\begin{array}{c}
\sin \frac{\theta}{2} \\
\cos \frac{\theta}{2}
\end{array}\right), \quad \nu_{A}=\left(\begin{array}{c}
-\sin \frac{\theta}{2} \\
\cos \frac{\theta}{2}
\end{array}\right),
$$

where the angle $\theta$ is that subtended by the copolar nulls on the Poincaré sphere. After a certain amount of manipulation and application of trigonometric doubling formulae, it is found that

$$
\frac{1+k}{1-k} \sin \theta=\gamma \sin \theta=\sin \psi(\cos \varphi+j \sin \varphi \cos \theta) .
$$

From this expression, when $\theta$ and $k$ are known then $\psi$ and $\varphi$ can be uniquely determined. From (38) it can be easily ascertain that as $k \rightarrow-1$, the value corresponding to a symmetric scattering matrix, the extent to which the angle $\psi$ of the axis of the corresponding pair $\chi^{A}, \rho^{A}$ is skewed from the symmetry axis of the copolar nulls tends uniformly to zero, the case of symmetric scattering matrix. It is also clear that for real $k$ the axis of corresponding pairs lies in the meridional plane. Also when $\frac{1+k}{1-k}$ is pure imaginary then the axis is skewed orthogonally to the meridional plane. The alternative definition of the bistatic Huynen fork therefore shares the same qualitative properties of that given by the conventional definition in terms of copolar powers, but has the nice property of being a unique descriptor, unchanged by reversal of the roles of transmit and receive antennas. Its geometric stability to path reversal can be attributed to the form of the cross-ratio. On reversing the path the scattering matrix is transposed and this is equivalent to taking the reciprocal of $k$. But this is simply equivalent to swapping $\chi^{A}$ and $\rho^{A}$ in (35). The same point is valid regarding the ordering of the copolar nulls, which is arbitrary. Thus, either transposition preserves the form of the alternative Huynen fork, and only changes the labeling.

\section{Simulations}

A significant challenge in making any sense of the modelling of bistatic scattering of targets is that a large number of parameters are potentially involved. In addition to the range of scattering geometries, one also has three Euler angles to consider for the orientation of the scatterer if it is not rotationally symmetric. In addition, one has to consider size and shape of the target. As a first step we considered dielectric Rayleigh 
ellipsoids as a compromise between simplicity and sufficiently interesting behaviour. They are also quite representative of elements of many natural targets, such as leaves and parts of cereal crops. This allows to consider quite wide ranges of basic shape, including cases of rotational symmetry within a single parametric framework. Although ellipsoids have three planes of symmetry, one can also investigate breaking that symmetry without difficulty, by creating offset superpositions of such matrices, albeit making a slightly unrealistic assumption of non-interaction between the adjacent scatterers. Such schemes have the advantage of being easily to model, and to replicate. In fact we found a sufficiently rich phenomenology just using ellipsoids, and linear combinations appeared to make little significant difference to the results.

The modelling of scattering was based on the presentation of van de Hulst where a small ellipsoidal scatterer can be described in terms of a polarizability tensor, whose diagonal elements are given (c.f. [44]) by

$$
\alpha_{i}=\frac{V}{4 \pi} \frac{\varepsilon_{r}-1}{1-L_{i}\left(\varepsilon_{r}-1\right)} \quad i=1,2,3
$$

where

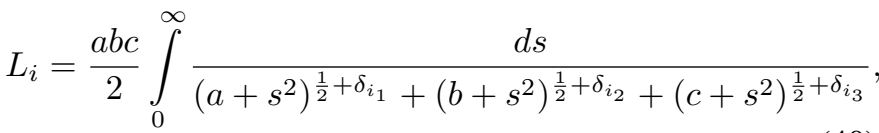

where $\varepsilon_{r}$ is the complex permittivity of the dielectric, $a, b$, $c$ are the ordered $(1,2,3)$ principal semi-axes of the ellipsoid and $\delta_{i j}$ is the Kronecker delta symbol. In general numerical methods are required to evaluate the integrals. In our case, they were evaluated symbolically in terms of elliptic integrals using Mathematica, after which numerical values were calculated. Van de Hulst [44] gives simple approximations that are reasonably accurate. Having obtained the polarizability tensor, the scattering matrix was found for any orientation of the scatterer by applying a 3D rotation to the tensor, and then evaluating the incident and far fields by application of the Green's dyadic, finally obtaining the scattering matrix by evaluating the voltages as functions of the transmit and antenna states. In the results presented, the scattering matrix is expressed relative to a common scattering plane, which reduces smoothly to the monostatic case.

\section{A. Results}

Simulations of scattering matrices were computed for different scatterers and different scattering geometries in order to explore the behaviour of $k$. The projective invariant $k$ characterises the bistatic scattering matrix which was associated with the conic using the corresponding polarizations states (see Sec. VI-A). The first type are simple Rayleigh ellipsoids of variable axial ratios and the second type are compound scatterers synthetised by superposition of two or more Rayleigh ellipsoids. Although it is just as easy to model general ellipsoids as spheroids, the parametric space becomes much larger. We have instead divided the results between spheroids on the one hand which we examine in some detail, and a synthetic case comprising offset spheroids which has no specific geometric symmetry. In the case of spheroids there is a rotational symmetry axis, and it is convenient to plot derived scalar invariants on a sphere where the position on the surface locates the pole of the symmetry axis of the scatterer as its orientation is changed with respect to a fixed bistatic geometry. The plots naturally have inversion symmetry. Only the bistatic opening angle is relevant to the form of the plot, so in Fig. 1523 the transmit and receive antennas are fixed in the $x y$ plane symmetrically with respect to the $x$ axis, while the bistatic angle may be varied. The plots are grouped according to shape and bistatic angle.

\section{B. Influence of scatterer shape}

Fig. 15 models an oblate spheroid with the ratio minor/major axis equal to 0.4 . The antennas pointing directions are $120^{\circ}$ apart, that is the bistatic angle is $\theta=120^{\circ}$. For pure

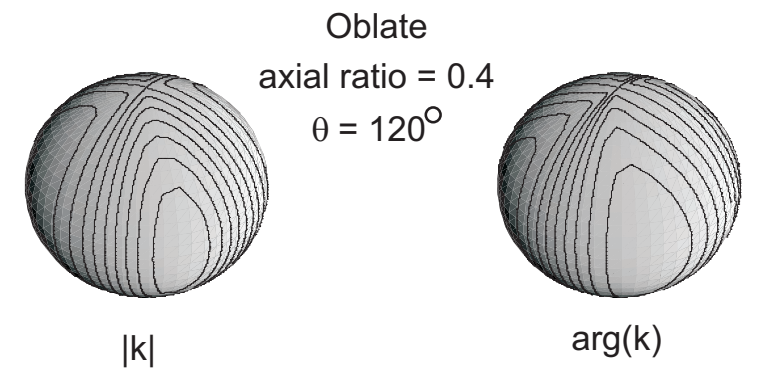

Fig. 15. Contour of the complex invariant $k$ as a function of the symmetry axis.

Rayleigh scatterers of homogeneous composition the value of the permittivity only affects the overall magnitude and phase, but not the ratios of the matrix elements. Plots of contours of the magnitude and phase of the $k$ invariant are displayed on the sphere of directions of the symmetry axis. Although there is some broad similarity between the pair of plots, there are also distinct differences. Fig. 16 shows the same data for a much flatter oblate spheroid, with minor/major ratio of 0.1 . Contours of the $k$-phase are concentrated more closely for

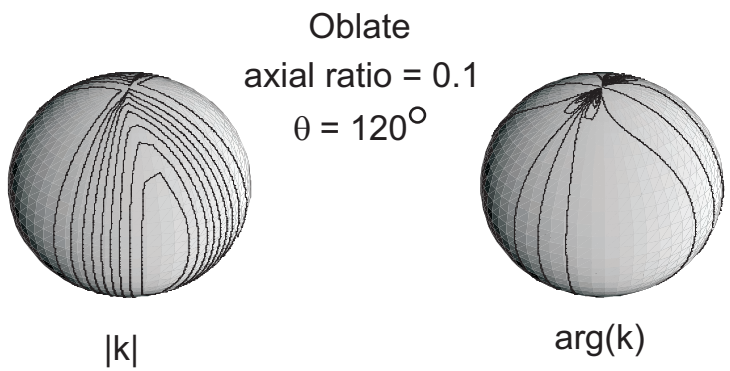

Fig. 16. Contour of the complex invariant $k$ as a function of the symmetry axis.

cases near where the maximal symmetry plane coincides with the plane of the antennas.

In contrast, Fig. 17 and 18 show prolate spheroids of the same composition as the oblate scatterers again with axial ratios of 0.4 and 0.1 . The plots show the same kind of symmetry in general, but the areas of relatively tight and sparse contours are different. 
Prolate

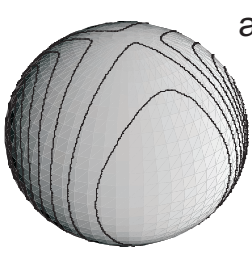

$|\mathrm{k}|$ axial ratio $=0.4$ $\theta=120^{\circ}$

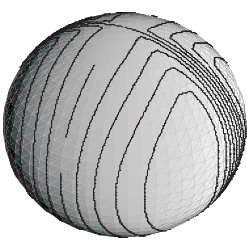

$\arg (\mathrm{k})$
Fig. 17. Contour of the complex invariant $k$ as a function of the symmetry axis.

\section{Prolate}

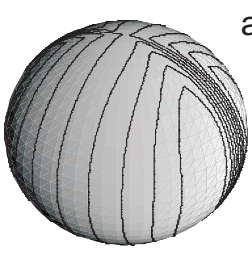

$|\mathrm{k}|$ axial ratio $=0.1$ $\theta=120^{\circ}$

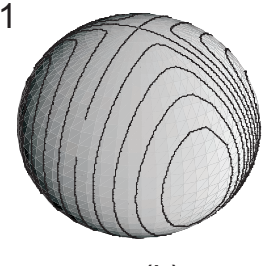

$\arg (\mathrm{k})$

Fig. 18. Contour of the complex invariant $k$ as a function of the symmetry axis.

\section{Dependence on bistatic angle}

We now consider variations of the bistatic angle for the cases of the moderate oblate and prolate scatterers. Firstly, Fig.

\section{Oblate}

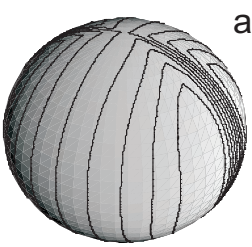

$|\mathrm{k}|$

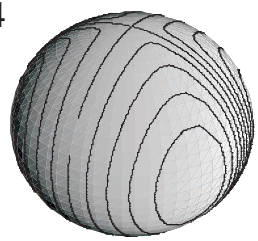

$\arg (\mathrm{k})$
Fig. 19. Contour of the complex invariant $k$ as a function of the symmetry axis.

19 shows the oblate scatterer of axis ratio 0.4 with a bistatic angle of $60^{\circ}$. Broadly the form of the plot is similar to the case of the wide bistatic angle, with the lobes of the contours closer together, broadly directed towards the two antennas. Fig. 20, however is the same scatterer with bistatic angle of $90^{\circ}$. The plot in this case is markedly dissimilar in form, showing quite intricate angular structure. Fig. 21 and 22 repeat the exercise for the prolate case, with similar results. This should perhaps not be surprising, since Rayleigh scattering even from spheres is polarimetrically very sensitive in this case.

\section{A model of a composite scatterer without intrinsic geomet- ric symmetry}

The second type of scatterer investigated here is of compound scatterers, synthesized by superposition of two or more Rayleigh ellipsoids, with arbitrary orientations and offsets

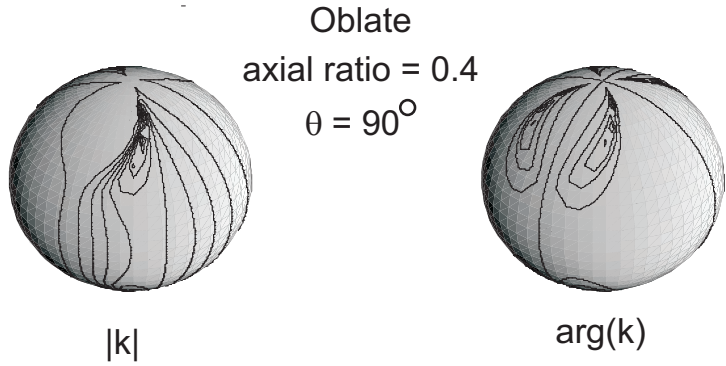

Fig. 20. Contour of the complex invariant $k$ as a function of the symmetry axis.

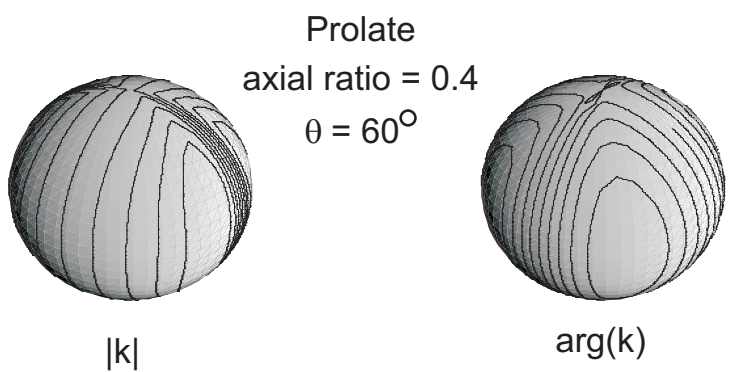

Fig. 21. Contour of the complex invariant $k$ as a function of the symmetry axis.

TABLE I

COMPOUND SCATTERER SPECIFICATION

\begin{tabular}{|l|l|l|}
\hline Element & $\mathbf{1}$ & $\mathbf{2}$ \\
\hline Relative Permittivity & $8.0-j 3.0$ & $2.5-j 1.5$ \\
\hline Shape & Oblate 0.1 & Prolate 0.5 \\
\hline Euler rotation (rad) & $-0.3,-0.8,0.4$ & $-0.3,0.5,0.7$ \\
\hline Centre offset (wavelengths) & $(0,0,0)$ & $(0.1,-0.18,0.05)$ \\
\hline Power weighting & 0.4 & 0.6 \\
\hline
\end{tabular}

from the origin. Here, there is freedom to vary the permittivity of the component parts, which gives a very large parametric space. There is no account taken for interaction between the elements, but in this way a scatterer without any symmetry can readily be constructed. For the scatterer detailed in Fig. 23, 24 and 25 the recipe for its construction is given in Table I.

In this case, there is no preferred orientation for the scatterer. The plots shown in Fig. 23 are superficially not greatly different from those for the pure ellipsoids. This may be due to one component dominating, or the resultant scatterer being reasonably well represented by an equivalent ellipsoid. As this case has no particular inherent symmetry, it is of interest to keep the scatterer fixed while varying the antenna geometry. Fig. 24 and 25 show the $k$-invariant as one antenna is fixed and the second one moved continuously from collocation (backscatter) to the forward scatter geometry. The plots show two interesting features. Firstly, it is clear that there is a critical angle near $90^{\circ}$ where the curves peak. In this case, the scatterer as a whole is not necessarily electrically small, so interference effects can play a part (as in Mie scatter). Also of 


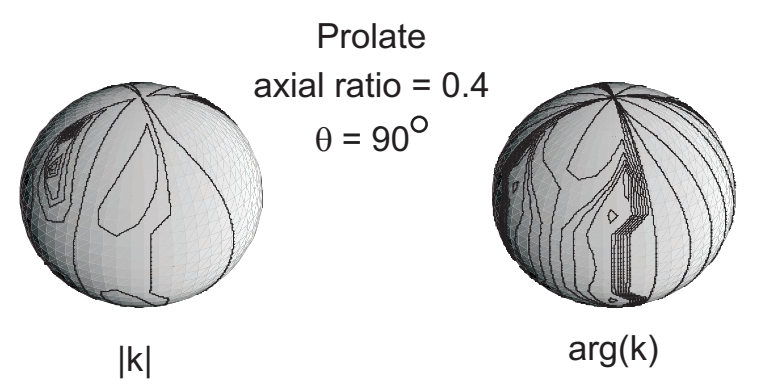

Fig. 22. Contour of the complex invariant $k$ as a function of the symmetry axis.

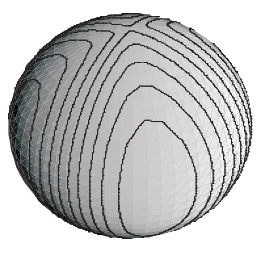

$|k|$
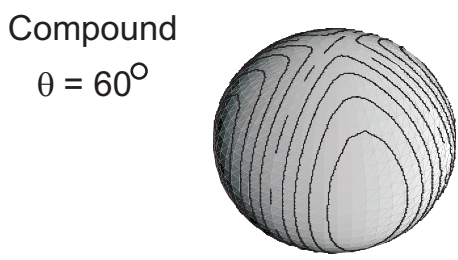

$\arg (\mathrm{k})$
Fig. 23. Contour of the complex invariant $k$ as a function of the symmetry axis.

note is the complementarity of the argument and phase of the $k$-invariant. For near backscatter, the amplitude is rather flat, but the phase of $k$ has a near linear variation. As the bistatic angle opens towards the forward scatter position, the opposite is the case. These conclusions were found to be generic for many randomly chosen examples using the same composition scheme.

In conclusion, the complex $k$-invariant shows sensitivity to scatterer shape and to the bistatic angle. While the modulus of the $k$-ratio turns out in many cases to be not the same as, though broadly similar to the ratios of the singular value decomposition, the complementarity of the complex argument of $k$, particularly in the case of near-backscatter configurations suggests that the new invariant characterization may well add to the capability to discriminate target features using invariant characterization.

\section{E. Comparison of the k-invariant with SVD}

It is not difficult to show that a general bistatic matrix, $S$, may be reduced by a unitary basis transformation up to some complex scaling factor to the form,

$$
S=\left(\begin{array}{cc}
\mu & \varepsilon \\
-\varepsilon & \mu^{-1}
\end{array}\right)
$$

with $\mu$ real and $\varepsilon$ complex. A Lorentz boost congruential transformation, which preserves projective invariance yields,

$$
\left(\begin{array}{cc}
\mu^{-\frac{1}{2}} & 0 \\
0 & \mu^{\frac{1}{2}}
\end{array}\right)\left(\begin{array}{cc}
\mu & \varepsilon \\
-\varepsilon & \mu^{-1}
\end{array}\right)\left(\begin{array}{cc}
\mu^{-\frac{1}{2}} & 0 \\
0 & \mu^{\frac{1}{2}}
\end{array}\right)^{T}=\left(\begin{array}{rr}
1 & \varepsilon \\
-\varepsilon & 1
\end{array}\right) .
$$

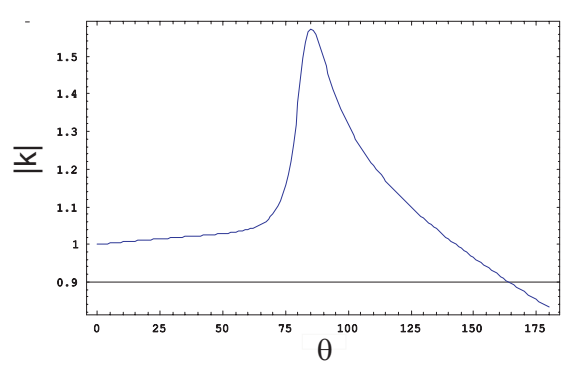

Fig. 24. The magnitude of $k$ for a compound scatterer for variable bistatic angles from backward to forward directions.

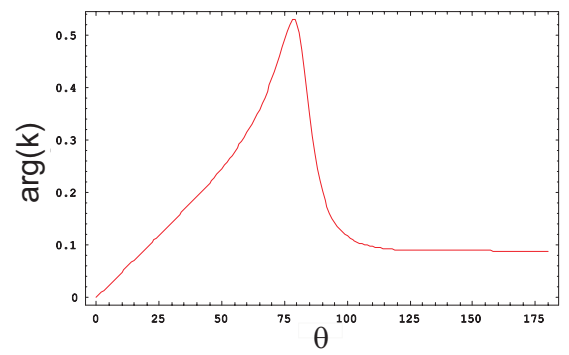

Fig. 25. The phase of $k$ for a compound scatterer for variable bistatic angles from backward to forward directions.

This may further be reduced to the homographic canonical form via,

$$
\frac{1}{2}\left(\begin{array}{ll}
1 & j \\
j & 1
\end{array}\right)\left(\begin{array}{cc}
\mu & \varepsilon \\
-\varepsilon & \mu^{-1}
\end{array}\right)\left(\begin{array}{ll}
1 & j \\
j & 1
\end{array}\right)^{T}=\left(\begin{array}{cc}
0 & \varepsilon+j \\
j-\varepsilon & 0
\end{array}\right)
$$

Hence the $k$-invariant of the scattering matrix is,

$$
k=\frac{\varepsilon-j}{\varepsilon+j}
$$

which is independent of $\mu$. The parameter is related to the Huynen angle $4 \gamma$ [27] between the copolar nulls of the scatterer (which is independent of the asymmetry, $\varepsilon$ ) by,

$$
2 \gamma=\arcsin \left(\frac{2 \mu}{1+\mu^{2}}\right) \text {. }
$$

For the singular decomposition of $S$, the amplitude ratio of the singular values can be determined by

$$
\mathrm{SVDR}^{2}=\frac{T+\sqrt{T^{2}-4 \Delta}}{T-\sqrt{T^{2}-4 \Delta}}
$$

where $T$ and $\Delta$ are respectively the trace and determinant of $S S^{\dagger}$ with superscript ${ }^{\dagger}$ denoting Hermitian conjugate. It is clear that logarithmically extreme values of SVDR occur when the determinant of $S$ vanishes, when $\varepsilon= \pm j$, and hence for the set $k=\{0, \infty\}$.

Since the $k-\varepsilon$ relation (44) is invertible, SVDR can be plotted as a function of the complex invariant $k$ and $\mu$ as real parameter. Since SVDR is invariant under sign change of $\varepsilon$, which in turn is equivalent to inversion of $k$, it is only necessary to plot for $k$ magnitudes less than unity. Fig. 26, 27 and 28 show SVDR as $\mu$ diverges progressively from the special case of unitary scatterers with antipodal copolar nulls. 


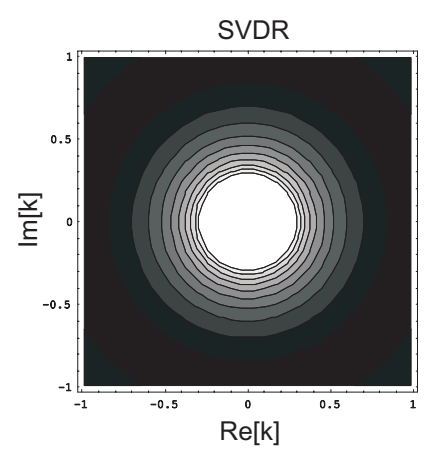

Fig. 26. SVDR contoured in the complex $k$ plane for $\mu=1$.

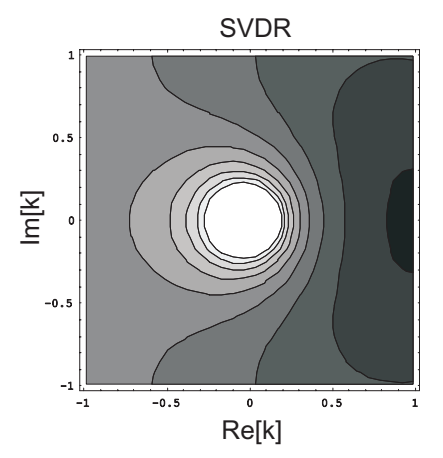

Fig. 27. SVDR contoured in the complex $k$ plane for $\mu=2$.

\section{Conclusions}

We have presented a new formulation for bistatic scattering founded on the principles of Geometric Polarimetry, in which (complex spinor) polarization states are geometrically represented by generators of the wave sphere. The scattering matrix is then determined by the geometry of an object in projective space that depends jointly on the scattering geometry and two characteristic polarizations. For every polarization of one antenna there is a corresponding one for the other for which the received voltage is null, and this relationship is geometrically determined. The formulation presented makes it possible to construct and analyse scattering configurations expressed solely in terms of antenna states, and this simplifies things practically by avoiding the need to consider both antenna and wave states (which have contragredient basis transformation laws) at the same time. Whilst two Hermitianorthogonal spinors may represent antiparallel directions, and hence a directional axis, the spin-frame normalization of a pair of spinors ensures that a rigid spatial reference frame is determined. In the context of bistatic scattering, this provides a robust method to ensure that the scattering plane and bisectrix are defined uniformly even in the degenerate case where the antenna pointing directions are parallel or antiparallel. In this scheme, path reversal, or exchange of antenna function between transmit and receive manifests itself solely in terms of transposal of the scattering matrix. In the special case of backscatter this is reflected naturally by the symmetry of the matrix. In the course of this work, a new complex scalar invariant was discovered. The complex $k$-invariant is related to the matrix asymmetry, but has been shown to be sensitive

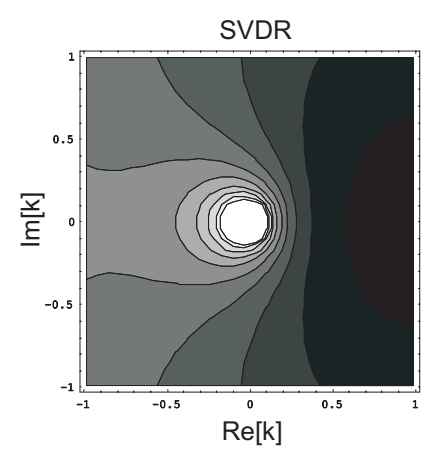

Fig. 28. SVDR contoured in the complex $k$ plane for $\mu=5$.

to scatterer shape as well as to the bistatic geometry, although $k=-1$ always in the backscatter case. Both the amplitude and phase of the $k$-invariant encode information about the scatterer, unlike the case of the singular value decomposition ratio, where only the magnitude is significant. We found a relationship between these invariants and the Huynen polarization fork angle for copolar nulls. From a practical point of view the fact that $k$ is projectively invariant rather than simply unitary invariant is of some interest. This means that $k$ is robust against non-unitary propagation transformations of congruential form. This does have limitations, however, because except in the backscatter case, propagation on the two paths between target and antennas is generally not the same. But if the propagation medium is shallow, and near the target, then for small bistatic angles the propagation effect on $k$ will be small even if there is polarization sensitive attenuation.

In conclusion, this paper, together with GP1 [21] provides a complete account of the 'classical' structure of polarimetry for general coherent scattering. Geometric Polarimetry has given a fully geometric interpretation to the voltage equation which expresses an invariant inner product between the wave field and the antenna height vector. The scattering matrix formulation in complex polarimetric notation expresses this in spinor form, the spinors for both wave and antenna states being reduced from the corresponding vector form via phase flags which are referred to the spin frame. Geometrically they are not vectors, but represent generators of the Poincare sphere. Basis transformations, and coherent processes in general, are Clifford translations [24] of the generators of one regulus of the Poincare sphere - they just shuffle them round the invariant sphere, also the sphere of normalized wave vectors [21]. To every coherent state there is a conjugate state, which is represented by a generator of the opposite regulus on the sphere. The concept of reality is preserved by always applying the conjugate of each transformation to the complementary regulus. We have striven to present Geometric Polarimetry in such a way that the externalities of radar polarimetry are almost untouched - one can still use matrix notation as long as quantities and their correct transformation laws are implicitly understood. By expressing the general voltage equation in terms of antenna states we retain the spirit of the IEEE standard for antennas [45]. What has changed is the inner interpretation, and it is this that allows polarimetry to be fully integrated in complexified geometry, rather than 
appear as it formerly has done, as an extraneous structure with seemingly idiosyncratic rules, especially the problematic history involving the handling of wave reversal.

This is evidently a very powerful approach. Since we have generalised the geometry to a projective framework, the structural nature of construction of relationships between points, line, and conics or quadrics does not change; but at the same time, problems relating to parallelism and infinite points are eliminated. Up to now, the geometry of complex Jones vectors has only been studied in relation to the plane of the wave front, whereas our complex projective picture reveals structurally invariant geometric relationships in bistatic scattering embedded in three spatial dimensions. This, we feel, is a significant and important conceptual development also because the geometric model provides a guarantee of invariant properties. Unavoidably, we have added a few new concepts such as the spin-frame and the phase flag, and the notion of corresponding states. These innovations are key, however. A spin frame has wider function than providing an orthogonal basis, for it defines a spatial reference frame. The phase flag determines, essentially, the angle at which the phase of a circularly polarized wave is zero. The notion of corresponding states provides the basis for a voltage equation expressed relative to a single spin frame in such a way that the backscatter alignment convention is an unexceptional special case.

A modified definition of the Huynen polarization fork agrees with the conventional one in the symmetric case, but is defined by adding to the usual copolar nulls a unique pair of orthogonal corresponding states; in other words, a pair for which the scattering amplitude between transmit and receive antennas is zero. This concept generalizes neatly from that of the symmetric form, which is not the case when one consider extremal values of copolar power. Thus, a unique tetrad of characteristic polarizations is determined by the structure of the symmetric part of the scattering matrix together with the new complex invariant $k$.

\section{ACKNOWLEDGMENT}

The work on Geometric Polarization was supported by the U.S. Office of Naval Research and the European Union (Marie Curie Research Training Network RTN AMPER, Contract number HPRN-CT-2002-00205). We also owe a debt of gratitude in particular to Prof. Wolfgang Boerner and the late Dr. Ernst Lueneburg, who were prime movers in stimulating the debate on the foundations of polarimetry, regardless of where that might lead.

\section{REFERENCES}

[1] A. Rosenqvist, M. Shimada, and M. Watanabe, "ALOS PALSAR: Technical outline and mission concepts," in 4th International Symposium of Bio- and Geophysical Parameters From SAR data for Land Applications, Innsbruck, Austria, Nov. 2004.

[2] A. Jameson and A. Kostinski, "Direct observations of coherent backscatter of radar waves in precipitation," Journal of the Atmospheric Sciences, vol. 67, pp. 3000-3005, 2010.

[3] J.-S. Lee and T. Ainsworth, "The effect of orientation angle compensation on coherency matrix and polarimetric target decompositions," IEEE Trans. on Geoscience and Remote Sensing, vol. 49, no. 1, pp. 53-64, 2011.
[4] R. Paladini, M. Martorella, and F. Berizzi, "Classification of manmade targets via invariant coherency-matrix eigenvector decomposition of polarimetric SAR/ISAR images," IEEE Trans. on Geoscience and Remote Sensing, vol. 49, no.8, pp. 3022-3034, 2011.

[5] M. Galletti, D. Bebbington, M. Chandra, and T. Borner, "Measurement and characterization of entropy and degree of polarization of weather radar targets," IEEE Trans. on Geoscience and Remote Sensing, vol. 46, no. 10 , pp. 3196-3207, 2008.

[6] H. Nies, F. Behrer, S. Reuter, O. Loffield, and R. Wang, "Polarimetric and interferometric applications in a bistatic hybrid SAR mode using TerraSAR-X," in Proc. of IGARSS 2010 - IEEE International Conference on Geoscience and Remote Sensing, Hawaii, U.S.A., July 2010.

[7] L. Guerriero, D. Pierdicca, L. Pulvirenti, and P. Ferrazzoli, "Use of satellite radar bistatic measurements for crop monitoring: A simulation study on corn," Remote Sensing, vol. 5(2), pp. 864-890, 2013.

[8] H. Carreno-Luengo, A. Amèzaga, D. Vidal, R. Olivé, J. Munoz, and A. Camps, "First polarimetric GNSS-R measurements from a stratospheric flight over boreal forests," Remote Sensing, vol. 7(10), pp. $13120-13138,2015$.

[9] V. S. del Rio, L. Abalde-Lima, and C. Christodoulou, "Electromagnetic scattering from vegetation cylindrical components," IEEE Geoscience and Remote Sensing Letters, vol. 12, no.4, pp. 751-755, 2015.

[10] S. Cloude and E. Pottier, "A review of target decomposition theorems in radar polarimetry," IEEE Trans. Geoscience and Remote Sensing, vol. 34, no.2, pp. 498-518, 1996.

[11] J.-L. Alvarez Perez, "Coherence, polarization, and statistical independence in Cloude-Pottier's radar polarimetry," IEEE Trans. Geoscience and Remote Sensing, vol. 49, no.1, pp. 426-441, 2011.

[12] M. Davidovitz and W.-M. Boerner, "Extension of Kennaugh's optimal polarization concept to the asymmetric scattering," IEEE Trans. on Antenna and Propagation, vol. 34, no.4, pp. 569-574, 1986.

[13] J. Huynen, "Phenomenological theory of radar targets," Ph.D. dissertation, Technical University of Delft, 1970.

[14] A.-L. Germond, E. Pottier, and J. Saillard, "Foundations of bistatic radar polarimetry theory," in Radar 97 (Conf. Publ. No. 449), 1997, pp. 833837.

[15] A.-L. Germond, "Théorie de la polarimetrie radar en bistatique," Ph.D. dissertation, Université de Nantes, 1999.

[16] S. Cloude, "On the status of bistatic polarimetry theory," in Proc. of IGARSS 2005 - IEEE International Conference on Geoscience and Remote Sensing, Seoul, Korea, Nov. 2005.

[17] L. Bombrun, "Extension of the target scattering vector model to the bistatic case," in Proc. of IGARSS 2010 - IEEE International Conference on Geoscience and Remote Sensing, Hawaii, U.S.A., July 2010.

[18] — "Bistatic polarimetric SAR in terms of roll-invariant parameters," in 5th Int. Workshop on Science and Applications of SAR Polarimetry (PolInSAR), Frascati, Italy, 2011.

[19] C. Titin-Schnaider, "Power optimization for polarimetric bistatic random mechanisms," IEEE Trans. on Geoscience and Remote Sensing, vol. 45, no.11, pp. 3646-3660, 2007.

[20] - "The physical meaning of bistatic polarimetric parameters," IEEE Trans. on Geoscience and Remote Sensing, vol. 48, no.5, pp. 2349-2356, 2010.

[21] D. Bebbington and L. Carrea, "Geometric Polarimetry - Part I: Spinors and wave states," IEEE Trans. Geoscience and Remote Sensing, vol. 52, no. 7, pp. 3908-3923, 2014.

[22] R. Jones, "A new calculus for the treatment of optical systems I: Description and discussion," J. Opt. Soc. Am., vol. 31, pp. 488-493, 1941.

[23] R. Penrose and W. Rindler, Spinors and Space-Time. Cambridge University Press, 1984, vol. 1.

[24] J. Semple and G. Kneebone, Algebraic Projective Geometry. Oxford: Clarendon Press, 1952.

[25] D. Bebbington and L. Carrea, "On mathematical and physical principles of transformations of the coherent radar backscatter matrix," IEEE Trans. Geoscience and Remote Sensing, vol. 50, no. 11, pp. 4657-4669, 2012.

[26] E. Lüneburg, "Aspects of radar polarimetry," Elektrik-Turkish Journal of Electrical Engineering and Computer Science, vol. 10, no.2, pp. 219243, 2002.

[27] J. Huynen, "Phenomenological theory of radar targets," in Electromagnetic Scattering, P. Uslenghi, Ed. Academic Press New York, 1978, pp. $653-712$.

[28] F. Wang, R. Guo, and Y. Huang, "Radar target recognition based on some invariant properties of the polarization scattering matrix," in Proc. of 2011 IEEE CIE International Conference on Radar, 2011, pp. 626629 
[29] C. Graves, "Radar polarization power scattering matrix," Proc. of I.R.E., vol. 44, pp. 248-252, February 1956.

[30] J. Hubbert, "A comparison of radar, optics, and specular null polarization theory," IEEE Trans. on Geoscience and Remote Sensing, vol. 32, no.3, pp. 658-671, 1994.

[31] S. Schelkunoff, "On diffraction and radiation of electromagnetic waves," Physical Review, vol. 56, no. 4, pp. 308-316, 1939.

[32] - Electromagnetic Waves. New York: D. Van Nostrand, 1943.

[33] G. Sinclair, "The transmission and reception of elliptically polarized waves," Proc. of IRE, vol. 38, pp. 148-151, 1950.

[34] H. Mott, Polarization in Antennas and Radar. John Wiley and Sons, 1986.

[35] A. Kostinski and W.-M. Boerner, "On the foundations of radar polarimetry," IEEE Trans. on Antenna and Propagation, vol. 34, pp. 1395-1404, 1986.

[36] H. Mieras, "Comments on 'On the foundations of radar polarimetry', by A.B. Kostinski and W-M. Boerner," IEEE Trans. on Antenna and Propagation, vol. 34, pp. 1470-1471, 1986.

[37] A. Kostinski and W.-M. Boerner, "Reply to 'Comments on On the foundations of radar polarimetry' by $\mathrm{H}$. Mieras," IEEE Trans. on Antenna and Propagation, vol. 34, pp. 1471-1473, 1986.

[38] J. Hubbert and V. Bringi, "Specular null polarization theory: Applications to radar meteorology," IEEE Trans. on Geoscience and Remote Sensing, vol. 34, no.4, pp. 859-873, 1996.

[39] E. Lüneburg, "Comments on the 'Specular null polarization theory', by J.C. Hubbert," IEEE Trans. on Geoscience and Remote Sensing, vol. 35, no. 4, pp. 1070-1071, 1997.

[40] J. Hubbert, "Reply to 'Comments on The specular null polarization theory', by E. Lüneburg," IEEE Trans. on Geoscience and Remote Sensing, vol. 35, no.4, pp. 1071-1072, 1997.

[41] D. Bebbington and L. Carrea, "On the response of an antenna to polarized electromagnetic plane waves using a tensorial and spinorial approach," in arxiv.org, 2013.

[42] M. Berry, "Quantal phase factors accompanying adiabatic changes," Proc. R. Soc. Lond., vol. A 392, pp. 45-57, 1984.

[43] D. Bebbington, L. Carrea, and G. Wanielik, "Application of geometric polarization to invariance properties in bistatic scattering," Adv. Radio Sci., vol. 3, pp. 421-425, 2005.

[44] H. V. der Hulst, Light Scattering by Small Particles. New York: Wiley, 1957.

[45] IEEE, Standard Definitions of Terms for Antennas (IEEE Std 145-1983), 1983.

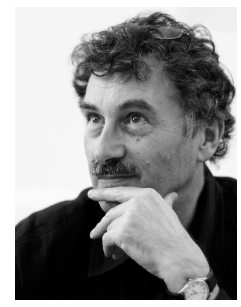

David Bebbington Received the B.A. degree in experimental and theoretical physics and the Ph.D. degree in radio astronomy from Cambridge University, Cambridge, U.K., in 1977 and 1986, respectively. From 1981 to 1984, he worked on millimeter wave propagation research at the Rutherford Appleton Laboratory. Since 1984, he has been at the University of Essex, Essex, U.K., and currently holds the post of Senior Lecturer in the Department of Computing and Electronic System. His interests are polarimetry, scattering, applications of wave propagation in remote sensing, and weather radars.

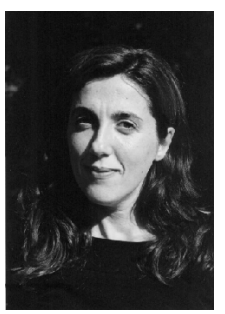

Laura Carrea received the Laurea degree in physics from the University of Turin, Italy in 1997 and her $\mathrm{Ph} . \mathrm{D}$. in Electronic Engineering from the University of Essex, U.K. in 2013. From 1999 to 2004 she was with the Chemnitz University of Technology, Germany partly with a Fellowship in the frame of the Marie Curie TMR Network "Radar Polarimetry: Theory and Application". From 2004 to 2013 she was with Centre for Remote Sensing \& Environmetrics at the University of Essex, U.K. and from 2014 with the Meteorology Dept. at the University of Reading, U.K. Her main interests include polarization and scattering for remote sensing and water surface temperature retrieval for climate studies. 